\title{
1 Protein-Metabolite Interactomics Reveals Novel Regulation of Carbohydrate Metabolism
}

3

5

Kevin G. Hicks¹, Ahmad A. Cluntun 1 , Heidi L. Schubert ${ }^{1}$, Sean R. Hackett², Jordan A. Berg ${ }^{1}$, Paul G. Leonard $^{3,4}$, Mariana A. Ajalla Aleixo ${ }^{5, \neq}$, Aubrie Blevins ${ }^{1, \neq}$, Paige Barta $^{1}$, Samantha Tilley ${ }^{1}$, Sarah Fogarty ${ }^{1,21}$, Jacob M. Winter ${ }^{1}$, Hee-Chul Ahn ${ }^{6,}$, , Karen N. Allen ${ }^{7,}$, , Samuel Block ${ }^{8,}$, Iara A. Cardoso ${ }^{5}$, Jianping Ding ${ }^{9,}$,

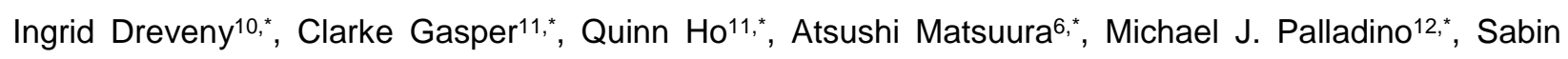

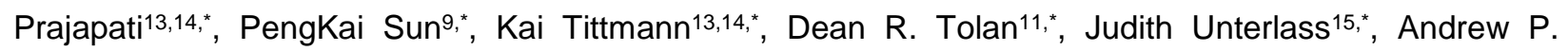

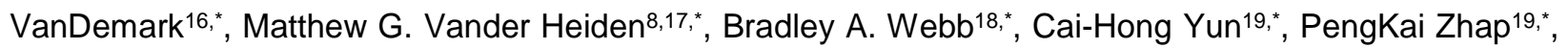
Christopher P. Hill' ${ }^{1}$, Maria Cristina Nonato ${ }^{5}$, Florian L. Muller ${ }^{20}$, Daniel E. Gottschling ${ }^{2}$, James E. Cox ${ }^{1}$, Jared Rutter ${ }^{1,21, \dagger}$

1Department of Biochemistry, University of Utah School of Medicine, Salt Lake City, UT 84112, USA.

${ }^{2}$ Calico Life Sciences LLC, South San Francisco, CA 94080, USA.

${ }^{3}$ Core for Biomolecular Structure and Function, University of Texas MD Anderson Cancer Center, Houston, TX 77054, USA.

${ }^{4}$ Institute for Applied Cancer Sciences, University of Texas MD Anderson Cancer Center, Houston, TX 77054, USA.

${ }^{5}$ Laboratório de Cristalografia de Proteinas, Faculdade de Ciências Farmacêuticas de Ribeirão Preto, Universidade de São Paulo, Ribeirão Preto, SP 14040-903, Brazil.

6Integrated Research Institute for Drug Development, College of Pharmacy, Dongguk University-Seoul, Goyang, Gyeonggi 10326, The Republic of Korea.

7Department of Chemistry, Boston University, Boston, MA 02215, USA.

${ }^{8}$ The Koch Institute for Integrative Cancer Research and Department of Biology, Massachusetts Institute of Technology, Cambridge, MA 02139, USA.

9State Key Laboratory of Molecular Biology, Shanghai Institute of Biochemistry and Cell Biology, Center for Excellence in Molecular Cell Science, University of Chinese Academy of Sciences, Shanghai, 200031, China.

${ }^{10}$ Biodiscovery Institute, School of Pharmacy, University of Nottingham, Nottingham, NG7 2RD, UK.

${ }^{11}$ Department of Biology, Boston University, Boston, MA 02215, USA.

${ }^{12}$ Department of Pharmacology \& Chemical Biology, University of Pittsburgh, Pittsburgh, PA 15261, USA.

${ }^{13}$ Department of Molecular Enzymology, Göttingen Center of Molecular Biosciences, University of Göttingen, Göttingen 37077, Germany.

${ }^{14}$ Department of Structural Dynamics, Max Planck Institute for Biophysical Chemistry, Göttingen 37077, Germany.

${ }^{15}$ Department of Oncology and Pathology, Karolinska Institute, Stockholm 171 76, Sweden. 
$37{ }^{16}$ Department of Biological Sciences, University of Pittsburgh, Pittsburgh, PA 15260, USA.

$38 \quad{ }^{17}$ Dana-Farber Cancer Institute, Boston, MA 02115 USA

$39{ }^{18}$ Department of Biochemistry, West Virginia University, Morgantown, WV 26506, USA.

40 19Department of Biophysics, School of Basic Medical Sciences, School of Basic Medical Sciences, Peking

41 University Health Science Center, Beijing 100191, China.

$42{ }^{20}$ Department of Cancer Systems Imaging, University of Texas MD Anderson Cancer Center, Houston, TX

4377054 , USA.

$44{ }^{21}$ Howard Hughes Medical Institute, University of Utah School of Medicine, Salt Lake City, Utah 84112, 45 USA.

46 *These authors are listed alphabetically.

$\ddagger$ Current addresses: Department of Molecular Biology, UT Southwestern Medical Center, Dallas, TX

75390, USA (AB); Brazilian Nanotechnology National Laboratory, Brazilian Center for Research in Energy and Materials, Campinas, SP 13083-970, Brazil (MAAA).

52 †Corresponding author email: rutter@biochem.utah.edu

\section{Abstract}

Metabolism is highly interconnected and also has profound effects on other cellular processes. However,

61 the interactions between metabolites and proteins that mediate this connectivity are frequently low affinity

62 and difficult to discover, hampering our understanding of this important area of cellular biochemistry.

63 Therefore, we developed the MIDAS platform, which can identify protein-metabolite interactions with

64 great sensitivity. We analyzed 33 enzymes from central carbon metabolism and identified 830 protein-

65 metabolite interactions that were mostly novel, but also included known regulators, substrates, products

66 and their analogs. We validated previously unknown interactions, including two atomic-resolution

67 structures of novel protein-metabolite complexes. We also found that both ATP and long-chain fatty acyl-

68 CoAs inhibit lactate dehydrogenase A (LDHA), but not LDHB, at physiological concentrations in vitro.

69 Treating cells with long-chain fatty acids caused a loss of pyruvate/lactate interconversion, but only in

70 cells reliant on LDHA. We propose that these regulatory mechanisms are part of the metabolic

71 connectivity that enables survival in an ever-changing nutrient environment, and that MIDAS enables a

72 broader and deeper understanding of that network. 


\section{Main Text}

Metabolites are the small molecule substrates, intermediates, and end products of metabolic pathways, and their physical interactions with proteins are among the most common and important interactions in biology (Fig. 1A). Metabolites are not only the chemical ingredients of metabolic reactions, but also enact enzyme regulation within and between metabolic pathways. Such regulatory interactions enable the maintenance of metabolic homeostasis in an environment where the availability and identity of nutrients are constantly changing. Despite their central importance, progress towards comprehensive identification of protein-metabolite interactions (PMIs) has been limited and sporadic. Unlike interactions involving other biological molecules, where we have robust and generalizable approaches such as coimmunoprecipitation for identifying protein-protein interactions (1) and chromatin immunoprecipitation for elucidating protein-DNA interactions (2,3), widely applicable strategies to detect PMls are lacking. Although some progress has been made recently (4), the very nature of many of these biologically important interactions presents a major hurdle to their identification. For example, to maximize the regulatory potential of protein binding, metabolites frequently interact with proteins with an affinity that is sufficiently weak to approximate their dynamic cellular concentrations_often high micromolar to low millimolar. Therefore, we developed the highly sensitive MIDAS (ㅆass spectrometry Integrated with equilibrium Dialysis for the discovery of $\underline{\text { Allostery }}$ Systematically) platform to enable the systematic discovery of PMls, including those low-affinity interactions that mediate cellular metabolic homeostasis.

The MIDAS platform is built on the biophysical principle of equilibrium dialysis (5) (Fig. 1B). A purified protein is separated from a defined library of metabolites by a semi-permeable dialysis membrane that allows passage of the metabolites but not the protein. After incubation, the system achieves relative equilibrium, such that the concentration of free (i.e. non-interacting) metabolites becomes similar on both sides of the membrane. However, the total concentration of those metabolites that interact with the protein is specifically elevated in the protein-containing chamber (Fig. 1B-purple triangles). The protein is then removed by precipitation and the relative metabolite abundance is quantified by high-throughput flow injection analysis mass spectrometry (FIA-MS). Interestingly, there are also metabolites that are selectively depleted from the protein-containing chamber (Fig. 1B-yellow stars), which likely results from enzymatic conversion or from very high affinity interactions including covalent protein modifications.

The MIDAS metabolite library comprises 401 compounds that together represent a sizable fraction of the water-soluble, stable, FIA-MS-detectable, and commercially available human metabolome (Fig. S1A and Data S1). Due to intrinsic differences in chemical structure and ionization properties, not all metabolites could be analyzed using the same FIA-MS parameters. We profiled each metabolite individually for its optimal FIA-MS polarity and mobile phase $\mathrm{pH}$ ionization and detection conditions (Data S2), and, guided by these criteria, we divided the library into four pools for multiplexed analysis (Fig. S1B and Data S1).

108 Next, we developed FIA-MS methods, optimized for each pool, that enabled rapid and robust quantitation 109 of the constituent metabolites. Splitting metabolites into pools also allowed us to separate some isomers so that they could be independently quantified, solving a technical shortcoming of FIA-MS. 
Having established the MIDAS methodology, we performed a pilot validation study using proteins

112 with well-established metabolite interactions. We analyzed three human proteins that converge to regulate 113 the mTORC1 kinase, an important nexus in growth factor signaling: CASTOR1, which binds arginine (6);

114 Sestrin2, which binds leucine, isoleucine, and methionine with decreasing affinity (7); and Rheb, which is a 115 GTPase that hydrolyses GTP to GDP (8) (Fig. S1C). In each case, the known ligands were the most 116 enriched and statistically significant interactors detected: CASTOR1 enriched arginine (Fig. 1C); Sestrin2 117 enriched the leucine/isoleucine/allo-isoleucine isomer group and methionine (Fig. 1D); and Rheb enriched 118 both its substrate, GTP, and its product, GDP, as well as the structually similar isomer group of ATP and 119 deoxyGTP (Fig. 1E). Thus, MIDAS effectively identified known PMIs, including regulators as well as enzyme 120 substrates and products.

121 The enzymes of glycolysis and related metabolic pathways are of particular interest for MIDAS 122 analysis due to their importance in almost all human cells and the extent of known metabolite interactions. 123 Therefore, we next used MIDAS to profile 33 human enzymes of central carbon metabolism, including 124 enzymes of glycolysis, gluconeogensis, the tricarboxylic acid (TCA) cycle, and the serine biosynthetic pathway that emerges from glycolysis (Fig. S1C). In total, we identified 830 putative PMls, the vast majority 126 of which were previously unknown (Table S3). Unsupervised hierarchical clustering (Fig. 2A) and 127 multidimensional scaling (Fig. 2E) of the entire PMI dataset demonstrated that structurally and functionally 128 related proteins frequently have very similar metabolite interactions. For example, phosphoglycerate 129 mutase (PGAM1/2), enolase (ENO1/2), fructose bisphosphatase (FBP1/2), and lactate dehydrogenase $130(\mathrm{LDHA} / \mathrm{B})$ isoforms all clustered closely together. However, this was not observed across all enzyme 131 isoforms nor would it be expected given the known role of enzyme isoforms to enable distinct biological 132 regulation of pathways in different contexts. The PK-M1 isoform of pyruvate kinase was noticeably different 133 from the PK-LR and PK-M2 isoforms, and the IDH2 and IDH3 isoforms of isocitrate dehydrogenase, which 134 catalyze similar chemistry but are evolutionarily and structurally unrelated (9), exhibited distinct metabolite 135 interactomes. Additionally, we observed clustering of multiple $N A D(H)$-dependent dehydrogenases: 136 glyceraldehyde-3-phosphate dehydrogenase (GAPDH), LDHA, LDHB, mitochondrial malate 137 dehydrogenase (MDH2), and 3-phosphoglycerate dehydrogenase (PHGDH). An analogous clustering of 138 structurally and functionally related metabolites was also apparent, including nicotinamide-containing 139 metabolites and flavin-adenine dinucleotide (Fig. 2B), phosphate-containing organic acids (Fig. 2C), and 140 several nucleotide monophosphates (Fig. 2D).

141 Analysis of the 830 putative PMls identified by the MIDAS platform showed that carbohydrates 142 were the predominant class of protein-interacting metabolite across central carbon metabolism (Fig. 2F). 143 This likely reflects both substrate/product relationships as well as the allosteric regulation of these enzymes, 144 which are largely involved in carbohydrate metabolism, by upstream or downstream metabolites (i.e., 145 feedforward and feedback regulation). The majority of non-carbohydrate PMls included amino acids, 146 nucleotides, and fatty acid derivatives. Such PMIs not only represent substrates and products of enzymes 147 in these pathways, but suggest both intra- and inter-pathway regulation of central carbon metabolism (Fig. 
2G). Notably, we also observed extensive interactions with metabolites outside of these pathways (Fig. $2 \mathrm{H})$. Together, these data likely illustrate the integration of local and distal metabolic information on central carbon metabolism, which provides intermediates for most biosynthetic pathways in the cell.

We next selected a subset of PMI datasets from individual proteins in central carbon metabolism

152 (see Fig. 2G) for deeper bioinformatic, biochemical and structural analyses. Enolase catalyzes the 153 penultimate step in glycolysis, and the most enriched and most statistically significant interactor for both 154 isoforms (ENO1 and ENO2) was phosphoserine (pSer, Fig. 3A). Intriguingly, pSer is an intermediate in the 155 serine biosynthetic pathway, which diverges from glycolysis just upstream of enolase. pSer is subsequently 156 converted into serine which in turn allosterically activates the M2 isoform of pyruvate kinase (10), the 157 enzyme immediately downstream of enolase in the glycolytic pathway. Differential scanning fluorimetry 158 (DSF), which measures the thermal stability of a target protein, showed that pSer (but not serine, 159 phosphotyrosine, or phosphate) robustly stabilized both ENO1 $(\mathrm{Kd}$ app $=1.38 \mathrm{mM})$ and ENO2 $(\mathrm{Kd}$ app $=1.15$ $160 \mathrm{mM}$ ) (Fig. 3B), similar to their substrate 2PG (Kdapp $=0.298 \mathrm{mM}$ and $0.289 \mathrm{mM}$, respectively). X-ray 161 crystallography of the pSer-ENO2 complex found that pSer asymmetrically bound to the ENO2 dimer at 162 one of the two active sites and partially overlapped with the 2PG phosphate binding site (Fig. 3C, D). 163 Furthermore, pSer promoted an "open" active site conformation relative to the substrate bound complex 164 observed as repositioning of loops 4 and 11 and alpha helices 7 and 11 (Fig. 3D). Surprisingly, pSer only 165 weakly inhibited enolase activity as measured in vitro (Fig. S2A), raising the intriguing possibility that this binding event might instead modulate other enolase activities such as one of the reported moonlighting functions $(11,12)$.

MIDAS identified 2-amino-3-phosphonopropionic acid (AP-3), a component of phosphonate metabolism (KEGG), as a putative interactor with fumarase, an enzyme in the tricarboxylic acid cycle that catalyzes the reversible hydration of fumarate to malate, which was also a significant hit (Fig. 3E). AP-3

172 fumarate $\left(\mathrm{Kd}_{\mathrm{app}}=3.87 \mathrm{mM}\right)$ (Fig. 3F). Kinetic assays demonstrated that AP-3 competitively inhibited 173 fumarase (Fig. S2B), and, consistent with this, the crystal structure of the complex revealed that AP-3 binds 174 in the active site of fumarase similarly to the known inhibitor citrate (Fig. 3G-H) (13). Although the 175 consequences of fumarase modulation by AP-3 in vivo are unclear, these findings demonstrate that MIDAS 176 can identify novel and functional protein-metabolite interations.

177 MIDAS datasets from additional proteins further confirmed the ability of MIDAS to identify known 178 interactions with substrates, products, and regulators: Glucose-6-phosphate isomerase (GPI) with its 179 substrates, glucose-6-phosphate and fructose-6-phosphate (hexose-6-phosphates) (Fig. 3I); 180 phosphofructokinase (PFKP) with its product (F-1,6BP/G-1,6BP) and a putative alternative substrate, 181 sedoheptulose-7-phosphate, (14) which is an intermediate in the pentose-phosphate pathway (Fig. 3J); 182 glyceraldehyde-3-phosphate dehydrogenase (GAPDH) with its substrate (NAD), and regulators (cyclicAMP, 183 creatine-phosphate, and malonyl-CoA) (15-17) (Fig. 3K); and the M2 isoform of pyruvate kinase (PK-M2) 184 with GDP and multiple amino acid regulators (18) (Fig. 3L). In every case, MIDAS also highlighted intriguing 
previously unknown interactions with distinct and non-overlapping sets of metabolites. For example, acylCoAs, inositol phosphates, nicotinamides, adenine nucleotides, and downstream glycolytic intermediates were found to interact with GPI (Fig. 3I). We also observed interactions between PFKP and several di- and triphosphate nucleotides, thiamine pyrophosphate, as well as with L-5-hydroxytryptophan, an intermediate in the conversion of tryptophan to serotonin (Fig. J). Ins(3,4,5)P3, 2,3-BPG, and HMG-CoA were identified as novel binding partners for GAPDH (Fig. 3K), while PK-M2 interacted with flavins, a folate, and a thyroid hormone intermediate (Fig. 3L). PK-M2 was previously reported to be allosterically regulated by thyroid hormone $T_{3}$, but the significance of this binding is unknown (19).

A comparison of MIDAS analyses for multiple isoforms of metabolic enzymes demonstrated both shared and unique metabolite interactions. Fructose bisphosphatase (FBP) catalyzes the conversion of fructose-1,6-bisphosphate to fructose-6-phosphate, a rate-limiting step in gluconeogenesis. Both isoforms (FBP1 and FBP2) interact with the known inhibitor AMP in addition to other nucleotide monophosphates. However, only FBP1 showed an interaction with glucosamine-6-phosphate, the rate-limiting intermediate in the hexosamine pathway, which emerges from fructose-6-phosphate (Fig. 3M). Similarly, isoforms of phosphoglycerate mutase (PGAM1 and PGAM2) interacted with a large set of metabolites, almost all of which were identical between them, with the exception of the soluble inositol phosphate inositol-1,3,4-P3 (PGAM1) and PIP2 and PIP3 (PGAM2) (Fig. 3N). This might reflect differential membrane recruitment and/or regulation of PGAM isoforms by phosphoinositide kinases, which are activated by growth factor signaling. These PMI data illustrate the power of MIDAS to enable the generation of hypotheses about potential novel regulatory events.

Lactate dehydrogenase (LDH) reversibly catalyzes the reduction of pyruvate to lactate coincident with the oxidation of NADH to NAD, a pivotal branchpoint in carbohydrate metabolism. Consumption of pyruvate by LDH competes with the mitochondrial uptake and oxidation by the TCA cycle to maximize ATP production. When mitochondrial pyruvate oxidation is limited, such as in hypoxia or aerobic glycolysis, LDH is critically important to regenerate NAD to enable continued glycolytic flux. Importantly, the LDH reaction is reversible and is also required to utilize lactate, a major circulating carbohydrate in mammals, as a fuel to support cellular functions (20). This firmly places LDH as a key node in carbohydrate metabolism.

MIDAS analysis of the two major isoforms of LDH, LDHA and LDHB, revealed interactions with

214 NADH and NAD and the structurally related nucleotides nicotinamide mononucleotide (NMN) and FAD, as 215 well as the competitive inhibitor, oxaloacetate (21), and other keto-acids related to the LDH substrates 216 lactate and pryuvate. We also observed two other classes of interacting metabolites, adenosine nucleotides 217 and free and acylated coenzyme A (Fig. 4A, B). To determine if either of these classes represent bona fide 218 PMls, we first used a thermal shift assay to measure the binding affinity of the three major adenosine 219 nucleotides, AMP, ADP, and ATP, for LDHA and LDHB, and compared to the cofactor NAD (Fig. 4C). ATP 220 interacted with both isoforms with a $\mathrm{Kd}_{\mathrm{app}}=0.636 \mathrm{mM}$ and $0.697 \mathrm{mM}$, respectively, which is a biologically 221 relevant affinity, given that the generally accepted intracellular steady state ATP concentration is $1-5 \mathrm{mM}$. 
222 The interactions of either LDH isoform with ADP and AMP may not be physiologically relevant given the 223 disparity between the Kd values for each interaction and the cellular concentrations of ADP and AMP ( 0.4 $224 \mathrm{mM}$ and $\sim 0.04 \mathrm{mM}$, respectively (22) (Fig. 4C). Enzymatic activity assays of the two LDH isoforms further 225 supported this conclusion as both AMP and ADP inhibited LDHA and LDHB only at supraphysiological 226 concentrations (Fig. 4D). Interestingly, despite similar binding affinities to both LDHA and LDHB (Fig. 4D), 227 ATP selectively inhibited only the LDHA isoform, with an $\mathrm{IC}_{50}$ of $2.3 \mathrm{mM}$. This discrepancy could potentially 228 relate to the opposing effects of ATP binding on the thermal stability of the two proteins (Fig. 4C). The 229 inhibition of LDHA by ATP appears to be competitive with NAD and lactate (Fig. S3A).

230 Next, we investigated the putative interaction between the LDH isoforms and coenzyme A (CoA) 231 or CoA conjugated to short, medium, or long-chain fatty acids (i.e, acyl-CoAs). Esterification of long-chain 232 (>12 carbons) fatty acids to $\mathrm{CoA}$ is required for their intracellular diffusion and transport into the 233 mitochondrial matrix where they undergo $\beta$-oxidation to produce ATP. The accumulation of these species 234 has been previously demonstrated to be a signal of carbon fuel excess (23). We observed that acyl-CoAs 235 inhibited LDHA as a function of fatty acid chain length. Neither CoA alone nor any acyl-CoA with a fatty acid 236 chain-length up to eight carbons affected enzyme activity, and C12:0-CoA only weakly inhibited LDHA, with 237 an $\mathrm{IC}_{50}>100 \mu \mathrm{M}$ (Fig. 4E). However, long-chain acyl-CoAs-C16:0-CoA (palmitoyl-CoA), C18:1-CoA 238 (oleoyl-CoA) and C20:0-CoA (arachidoyl-CoA), none of which are in the MIDAS library-were all potent 239 inhibitors of LDHA, with $I_{50}$ values of $\sim 1 \mu \mathrm{M}$ (Fig. 4E). The inhibition of LDHA by palmitoyl-CoA is non240 competitive with respect to both NAD and lactate, suggesting that it is binding to LDHA outside of the active site (Fig. S3B). Intriguingly, LDHB, which shares $85 \%$ amino acid sequence identity with LDHA, was completely impervious to all tested acyl-CoAs, even at concentrations up to $100 \mu \mathrm{M}$ (Fig. 4F).

Having observed that palmitoyl-CoA inhibited LDHA, but not LDHB, we employed two orthogonal approaches to test for a physical interaction. Using a thermal stability assay, we found that low micromolar concentrations of palmitoyl-CoA, similar to the $\mathrm{IC}_{50}$, induced the formation of a distinct thermo-labile species of LDHA, while inducing a thermo-stable species of LDHB (Fig. S3C). These data indicate that LDHA and

247 LDHB both directly interact with palmitoyl-CoA with a physiologically relevant low micromolar affinity. Next, 248 we found that purified LDHA and LDHB bound to palmitoyl-CoA immobilized on agarose beads, and that 249 the binding for either protein was disrupted by elution with palmitoyl-CoA but not with buffer or acetyl-CoA 250 (C2:0-CoA) (Fig. S3D).

251 Given that palmitoyl-CoA inhibited LDHA at concentrations within the physiological range, we next 252 tested whether this inhibition is relevant in intact cells. We performed metabolic tracing experiments using 253 H9c2 rat cardiomyoblasts in which we knocked out Ldha, Ldhb, or both (Fig. S3E). We treated cells with $254{ }^{13} \mathrm{C}$-labeled glucose in the presence or absence of BSA-palmitate, which allows for efficient delivery of the 255 fatty acid into the cell where it can esterified to palmitoyl-CoA (Fig. 4G). We then used mass spectrometry 256 to measure the uptake and assimilation of ${ }^{13} \mathrm{C}$ into lactate. All four cell lines (WT, Ldha-/-, Ldhb-/- and Ldha257 /- Ldhb-/-) showed a similar ( 80\%) increase in intracellular palmitate following incubation with its BSA258 conjugate (Fig. S3F). Importantly, palmitate decreased the labelling of lactate from ${ }^{13} \mathrm{C}$-glucose, but only in 
wild-type (WT) and Ldhb-/- cells i.e., in cells in which LDHA is still present (Fig. 4H, S3G-H), implying that palmitate inhibition of glucose-to-lactate conversion is completely dependent upon LDHA in this cell line. This result indicates that one or more steps between glucose uptake and LDHA-catalyzed lactate synthesis is inhibited by palmitate treatment. To probe more specifically the uptake and LDH-mediated oxidation of lactate to pyruvate, we performed analogous experiments with ${ }^{13} \mathrm{C}$-lactate (Fig. 4I). Again, we found that treatment with palmitate inhibited the generation of ${ }^{13} \mathrm{C}$-pyruvate in WT and LDHB-/- cells, but pyruvate labeling in Ldha-/- or Ldha-/- Ldhb-/- cells was unaffected (Fig. 4J, S3I-J). These data suggest a novel mode of regulatory crosstalk between fatty acid and carbohydrate metabolism.

It is intriguing that we found that both ATP and long-chain acyl-CoAs preferentially inhibit LDHA, but not LDHB. LDHA and LDHB, the two dominant isoforms of lactate dehydrogenase, are expressed in a tissue-specific pattern such that the liver almost exclusively expresses LDHA, while the heart has high expression of LDHB (Fig. S4A, B). Importantly, the $\mathrm{IC}_{50}$ for inhibition by ATP is easily within the range of normal intracellular ATP concentrations, suggesting that LDHA might be partially inhibited in all cells with normal energy status. Given that the liver, the most LDHA-dominant tissue, is capable of catabolizing multiple substrates, inhibition by ATP might be a mechanism to spare carbohydrates for those cell types that are dependent upon them. This is particularly important given the recent demonstration that lactate may be a major carbohydrate fuel consumed by some tissues (20). Likewise, our observation that longchain acyl-CoAs inhibit LDHA could explain a previously described physiological phenomenon wherein fatty acids, released from the adipose tissue during fasting, inhibit lactate production and increase glucose production in the liver (24). We hypothesize that fatty acyl-CoA-mediated inhibition of LDHA would redirect pyruvate toward gluconeogensis and away from excretion following its conversion to lactate. Another potential implication of these results lies in the substantial interest in LDHA-specific inhibitors to block aerobic glycolysis in cancers $(25,26)$. Perhaps the mechanism(s) employed by ATP and acyl-CoAs could be exploited therapeutically.

This fatty acid-carbohydrate inter-pathway metabolic regulation is just one potential example of the

284 myriad metabolite-level regulatory events that enforce organismal homeostasis, which is vital to appropriately respond to stressors such as the feed-fast cycle, exercise, and infection. We propose that interactions between proteins and metabolites mediate much of this control. We have validated MIDAS as a robust platform for the discovery of these critical mechanisms, particularly for the detection of very low

288 affinity interactions, which include most of those involving high abundance cellular metabolites. In addition to recent discoveries of functionally important PMIs (27-29), MIDAS now identified hundreds of known and novel putative interactions with the enzymes of central carbon metabolism. Together, these serve as a roadmap for identifying new modes of metabolic regulation as well as previously undescribed alternative

292 substrates. We propose that the comprehensive discovery of such interactions will revolutionize our 293 understanding of cell biology and that MIDAS can empower a renewed focus on this challenging and critical 294 important area of biology. 


\section{Acknowledgements}

297 We thank members of the Rutter lab for helpful discussions and comments on the manuscript. We thank

298 Roche and Navitor for providing proteins for MIDAS analyses. We thank the University of Utah Mutation

299 Generation and Detection Core for providing CRISPR reagents, cell genotyping services and construction

300 of bacterial expression vectors. We thank the University of Utah Drug Discovery Core Facility for

301 generating the Ldha and Ldhb mutant cells. Metabolomics analysis was performed in the Metabolomics

302 Core Facility at the University of Utah. Mass spectrometry equipment was obtained through NCRR

303 Shared Instrumentation Grant 1S10OD016232-01, 1S10OD018210-01A1 and 1S10OD021505-01. This

304 work was supported by T32DK091317 and T32DK007115 to KGH, U54DK110858, R35GM131854, and a

305 grant from Calico Life Sciences, LLC to JR. JR is an investigator of the Howard Hughes Medical Institute.

$306 \mathrm{KGH}$ and JR are inventors of MIDAS technology that has been licensed to Atavistik Bio, for which KGH is

307 a consultant and JR is a founder.

\section{References}

310 1. E. L. Huttlin et al., The BioPlex Network: A Systematic Exploration of the Human Interactome. Cell $311 \quad 162,425-440(2015)$.

312 2. D. S. Gilmour, J. T. Lis, Detecting protein-DNA interactions in vivo: distribution of RNA polymerase 313 on specific bacterial genes. Proc Natl Acad Sci U S A 81, 4275-4279 (1984).

3143 3. D. S. Gilmour, J. T. Lis, In vivo interactions of RNA polymerase II with genes of Drosophila 315 melanogaster. Mol Cell Biol 5, 2009-2018 (1985).

3164 4. M. Diether, U. Sauer, Towards detecting regulatory protein-metabolite interactions. Curr Opin $317 \quad$ Microbiol 39, 16-23 (2017).

318 5. T. Orsak et al., Revealing the allosterome: systematic identification of metabolite-protein interactions. Biochemistry 51, 225-232 (2012).

6. L. Chantranupong et al., The CASTOR Proteins Are Arginine Sensors for the mTORC1 Pathway.

322 7. R. L. Wolfson et al., Sestrin2 is a leucine sensor for the mTORC1 pathway. Science 351, 43-48 323 (2016).

324 8. K. Inoki, Y. Li, T. Xu, K. L. Guan, Rheb GTPase is a direct target of TSC2 GAP activity and regulates 325 mTOR signaling. Genes Dev 17, 1829-1834 (2003).

3269 9. P. Sun, Y. Liu, T. Ma, J. Ding, Structure and allosteric regulation of human NAD-dependent 327 isocitrate dehydrogenase. Cell Discov 6, 94 (2020).

328 10. B. Chaneton et al., Serine is a natural ligand and allosteric activator of pyruvate kinase M2. Nature 491, 458-462 (2012).

330 11. R. Lopez-Alemany et al., Inhibition of cell surface mediated plasminogen activation by a monoclonal antibody against alpha-Enolase. Am J Hematol 72, 234-242 (2003). 
332 12. S. Feo, D. Arcuri, E. Piddini, R. Passantino, A. Giallongo, ENO1 gene product binds to the c-myc promoter and acts as a transcriptional repressor: relationship with Myc promoter-binding protein 1 (MBP-1). FEBS Lett 473, 47-52 (2000).

13. T. Weaver, L. Banaszak, Crystallographic studies of the catalytic and a second site in fumarase C from Escherichia coli. Biochemistry 35, 13955-13965 (1996).

14. K. Nakahigashi et al., Systematic phenome analysis of Escherichia coli multiple-knockout mutants reveals hidden reactions in central carbon metabolism. Mol Syst Biol 5, 306 (2009).

15. S. Galvan-Pena et al., Malonylation of GAPDH is an inflammatory signal in macrophages. Nat Commun 10, 338 (2019).

16. M. Nakano, S. Funayama, M. B. de Oliveira, S. L. Bruel, E. M. Gomes, D-glyceraldehyde-3phosphate dehydrogenase from HeLa cells--1. Purification and properties of the enzyme. Comp Biochem Physiol B 102, 873-877 (1992).

18. M. Yuan et al., An allostatic mechanism for M2 pyruvate kinase as an amino-acid sensor. Biochem

17. M. Oguchi, E. Gerth, B. Fitzgerald, J. H. Park, Regulation of glyceraldehyde 3-phosphate J 475, 1821-1837 (2018).

19. K. Ashizawa, P. McPhie, K. H. Lin, S. Y. Cheng, An in vitro novel mechanism of regulating the activity of pyruvate kinase M2 by thyroid hormone and fructose 1, 6-bisphosphate. Biochemistry 30, 7105-7111 (1991).

20. S. Hui et al., Glucose feeds the TCA cycle via circulating lactate. Nature 551, 115-118 (2017).

21. E. K. Wiese et al., Enzymatic activation of pyruvate kinase increases cytosolic oxaloacetate to inhibit the Warburg effect. Nat Metab 3, 954-968 (2021).

22. G. J. Gowans, D. G. Hardie, AMPK: a cellular energy sensor primarily regulated by AMP. Biochem Soc Trans 42, 71-75 (2014).

23. T. R. Koves et al., Mitochondrial overload and incomplete fatty acid oxidation contribute to skeletal muscle insulin resistance. Cell Metab 7, 45-56 (2008).

24. D. K. Sindelar et al., The role of fatty acids in mediating the effects of peripheral insulin on hepatic glucose production in the conscious dog. Diabetes 46, 187-196 (1997).

25. J. Billiard et al., Quinoline 3-sulfonamides inhibit lactate dehydrogenase A and reverse aerobic glycolysis in cancer cells. Cancer Metab 1, 19 (2013).

26. G. Rai et al., Discovery and Optimization of Potent, Cell-Active Pyrazole-Based Inhibitors of Lactate Dehydrogenase (LDH). J Med Chem 60, 9184-9204 (2017).

27. G. A. Bezerra et al., Crystal structure and interaction studies of human DHTKD1 provide insight into a mitochondrial megacomplex in lysine catabolism. IUCrJ 7, 693-706 (2020). 
28. G. A. Bezerra et al., Identification of small molecule allosteric modulators of 5,10methylenetetrahydrofolate reductase (MTHFR) by targeting its unique regulatory domain. Biochimie 183, 100-107 (2021).

29. Q. Hao et al., Sugar phosphate activation of the stress sensor elF2B. Nat Commun 12, 3440 (2021).

30. J. D. Storey, R. Tibshirani, Statistical significance for genomewide studies. Proc Natl Acad Sci U S A 100, 9440-9445 (2003).

31. F. H. Niesen, H. Berglund, M. Vedadi, The use of differential scanning fluorimetry to detect ligand interactions that promote protein stability. Nat Protoc 2, 2212-2221 (2007).

32. N. Satani et al., ENOblock Does Not Inhibit the Activity of the Glycolytic Enzyme Enolase. PLoS One 11, e0168739 (2016).

33. T. G. Battye, L. Kontogiannis, O. Johnson, H. R. Powell, A. G. Leslie, iMOSFLM: a new graphical

34. P. Evans, Scaling and assessment of data quality. Acta Crystallogr D Biol Crystallogr 62, 72-82 (2006).

35. P. R. Evans, G. N. Murshudov, How good are my data and what is the resolution? Acta Crystallogr D Biol Crystallogr 69, 1204-1214 (2013).

36. A. J. McCoy et al., Phaser crystallographic software. J Appl Crystallogr 40, 658-674 (2007).

37. D. Liebschner et al., Macromolecular structure determination using X-rays, neutrons and electrons: recent developments in Phenix. Acta Crystallogr D Struct Biol 75, 861-877 (2019).

38. P. Emsley, B. Lohkamp, W. G. Scott, K. Cowtan, Features and development of Coot. Acta Crystallogr D Biol Crystallogr 66, 486-501 (2010).

40. W. Kabsch, Xds. Acta Crystallogr D Biol Crystallogr 66, 125-132 (2010).

41. A. Vagin, A. Teplyakov, Molecular replacement with MOLREP. Acta Crystallogr D Biol Crystallogr 66, 22-25 (2010).

42. G. N. Murshudov, A. A. Vagin, E. J. Dodson, Refinement of macromolecular structures by the maximum-likelihood method. Acta Crystallogr D Biol Crystallogr 53, 240-255 (1997).

43. C. J. Williams et al., MolProbity: More and better reference data for improved all-atom structure validation. Protein Sci 27, 293-315 (2018).

399 44. A. Xiao et al., CasOT: a genome-wide Cas9/gRNA off-target searching tool. Bioinformatics 30, 1180-1182 (2014).

45. A. A. Cluntun et al., The rate of glycolysis quantitatively mediates specific histone acetylation sites. Cancer Metab 3, 10 (2015). 
403 46. M. J. Lukey et al., Liver-Type Glutaminase GLS2 Is a Druggable Metabolic Node in Luminal$404 \quad$ Subtype Breast Cancer. Cell Rep 29, 76-88 e77 (2019).

405 47. A. A. Cluntun et al., The pyruvate-lactate axis modulates cardiac hypertrophy and heart failure. Cell $406 \quad$ Metab 33, 629-648 e610 (2021).

407 48. J. M. Buescher et al., A roadmap for interpreting (13)C metabolite labeling patterns from cells. Curr Opin Biotechnol 34, 189-201 (2015).

409 49. J. Qin, G. Chai, J. M. Brewer, L. L. Lovelace, L. Lebioda, Structures of asymmetric complexes of human neuron specific enolase with resolved substrate and product and an analogous complex with two inhibitors indicate subunit interaction and inhibitor cooperativity. J Inorg Biochem 111, 187194 (2012). 


\section{Materials and Methods}

414 MIDAS metabolite library construction and storage

415 The MIDAS metabolite library (Fig. S1A) was constructed by extracting and cross-referencing primary and 416 secondary metabolites from KEGG and HMDB, with a focus on endogenous and exogenous compounds 417 that were quantified, detected, or predicted in human metabolism. All metabolites used in this study were 418 purchased from Sigma-Aldrich, Cayman Chemicals, Avanti Polar Lipids, Enamine, Combi-Blocks, Inc, or 419 custom sourced using Aldrich Market Select (Data S1). Metabolites were solvated to $10 \mathrm{mM}$ in molecular 420 grade water (Sigma-Aldrich W4502) or DMSO (Sigma-Aldrich D1435) and, where necessary to increase 421 solubility, titrated with acid or base. The MIDAS metabolite library was arrayed $1 \mathrm{~mL}$ per well in 96-deep 422 well storage plates (Greiner 780280), sealed with aluminum foil seals (VWR 60941-112), and stored at -

$42380^{\circ} \mathrm{C}$. When working stocks were needed, metabolites were moved from the deep well storage plates and 424 arrayed, $50 \mu \mathrm{L}$ per well, across multiple, single-use 384-well small volume plates storage plates (Greiner 425 781280), sealed with aluminum foil seals (VWR 60941-112), and stored at $-80^{\circ} \mathrm{C}$. Metabolite library 426 management and manipulation was conducted on a Beckman Coulter Biomek NXp SPAN-8 liquid handling 427 robot.

MIDAS metabolite library validation and pooling

430 Metabolite accurate mass, adduct, ionization, and detection parameters were determined using a flow 431 injection analysis mass spectrometry (FIA-MS) scouting approach to design four defined metabolite 432 screening pools (Fig. S1B) (Data S2). Briefly, 20 pmol of each metabolite from the MIDAS metabolite library 433 was independently assayed in positive and negative mode in technical quadruplicate $1 \mu \mathrm{L}$ injections with 434 interspersed blank injections by FIA-MS on a binary pump Agilent 1290 Infinity UHPLC system operated 435 with a flow rate of $0.1 \mathrm{~mL} / \mathrm{min}$ coupled to an Agilent 6550 ESI-QTOF MS. The following mobile phases were 436 used for FIA-MS scouting: $20 \mathrm{mM}$ formic acid pH 3 (Sigma-Aldrich F0507), $10 \mathrm{mM}$ ammonium acetate $\mathrm{pH}$ 4375 (Sigma-Aldrich 73594), $10 \mathrm{mM}$ ammonium acetate $\mathrm{pH} 6.8$ (Sigma-Aldrich 73594), and $10 \mathrm{mM}$ ammonium 438 bicarbonate $\mathrm{pH} 9$ (Sigma-Aldrich 09830). Source conditions consisted of $250^{\circ} \mathrm{C}$ gas temp, $11 \mathrm{~L} / \mathrm{min}$ gas 439 flow, 20 psig nebulizer, $400^{\circ}$ sheath gas temperature, $12 \mathrm{~L} / \mathrm{min}$ sheath gas flow, and $2000 \mathrm{~V}$ nozzle voltage. 440 Agilent MassHunter 7 software was used to qualitatively validate and quantify metabolites. The optimal 441 signal for each metabolite was determined by integrating the area under the curve of the extracted ion 442 chromatogram (XIC-AUC) for each metabolite adduct at the various mobile phase $\mathrm{pH}$ and instrument 443 polarity. The optimal adduct, $\mathrm{pH}$, polarity, metabolite solvent, and, if necessary, isomer family of each 444 metabolite was considered to construct four unique and defined MIDAS metabolite screening pools (Data 445 S1).

MIDAS protein-metabolite screening

448 The day of MIDAS screening, a number of MIDAS metabolite library, 384-well small volume working stock 449 plates, corresponding to the number of proteins to be screened (eight proteins per plate), were defrosted 
at $30^{\circ} \mathrm{C}$ for 5 minutes and metabolites were combined de novo to generate four predetermined MIDAS screening pools (Data S1). The MIDAS screening pools were prepared in LC-MS grade $150 \mathrm{mM}$ ammonium 452 acetate $\mathrm{pH} 7.4$ (Sigma-Aldrich 73594) and pH-adjusted with ammonium hydroxide (Sigma-Aldrich 338818).

453 The majority of metabolites were prepared to a final screening concentration of $50 \mu \mathrm{M}$ in the metabolites

454 pools, with a subset at higher or lower concentration dependent on their FIA-MS ionization properties (Data 455 S1). For each metabolite pool, $8 \mu \mathrm{L}$ of target protein (Fig. S1C and Data S3) was arrayed in a minimum of 456 a triplicate across a $10 \mathrm{kDa}$ MWCO 96-well microdialysis plate (SWISSCI Diaplate ${ }^{\mathrm{TM}}$ ) and sealed with 457 aluminum foil seals (Beckman Coulter 538619) to create the protein chambers. To the reverse side, 300

$458 \mu \mathrm{L}$ of metabolite pool was aliquoted per target protein replicate and sealed with aluminum foil seals 459 (Beckman Coulter 538619) to create the metabolite chambers. Where necessary and just prior to screening, 460 proteins provided in alternative buffer systems were in situ, sequentially exchanged into $150 \mathrm{mM}$ ammonium 461 acetate pH 7.4 (Sigma-Aldrich 73594) on the 96-well microdialysis screening plate (SWISSCI Diaplate ${ }^{\mathrm{TM}}$ ). 462 Loaded dialysis plates were placed in the dark at $4^{\circ} \mathrm{C}$ on a rotating shaker $(120 \mathrm{rpm})$ and incubated for 40 463 hours. Post-dialysis, protein and metabolite chamber dialysates were retrieved, sample volume normalized 464 and diluted 1:10 in 80\% methanol (Sigma-Aldrich 1060351000) to precipitate protein, incubated 30 mins on 465 ice, and centrifuged at $3200 \times \mathrm{g}$ for 15 mins to sequester precipitated protein. Processed protein and 466 metabolite chamber dialysates were retrieved and arrayed across a 384-well microvolume plate (Thermo 467 Scientific AB-1056), sealed with a silicon slit septum cap mat (Thermo Scientific AB-1171), and placed at $4^{\circ} \mathrm{C}$ for FIA-MS analysis.

MIDAS flow injection analysis mass spectrometry analysis

All MIDAS metabolite pool FIA-MS was performed on a Shimadzu Nexera HPLC system equipped with binary LC-20ADXR pumps and a SIL-20ACXR autosampler coupled to a SCIEX X500R ESI-QTOF MS.

473 Briefly, $2 \mu \mathrm{L}$ of each processed protein and metabolite chamber dialysate ( 10 pmoles per metabolite, 474 depending on metabolite) was injected in technical triplicate with blanks injections interspersed between 475 technical replicates. Mobile phase flow rate was $0.2 \mathrm{~mL} / \mathrm{min}$. The following mobile phases were used 476 according to the MIDAS metabolite pool being analyzed: pool 1, $5 \mathrm{mM}$ ammonium acetate pH 5 (Sigma477 Aldrich 73594), 50\% methanol (Honeywell LC230-4); pools 2 and 4, $5 \mathrm{mM}$ ammonium acetate pH 6.8 478 (Sigma-Aldrich 73594), 50\% methanol (Honeywell LC230-4); pool 3, $10 \mathrm{mM}$ formic acid pH 3 (Sigma479 Aldrich F0507), 50\% methanol (Honeywell LC230-4). Pools 1 and 2 were analyzed in positive mode and 480 pools 3 and 4 were analyzed in negative mode. Source conditions consisted of 40 psi for ion source gas 1 481 and 2,30 psi curtain gas, $600^{\circ} \mathrm{C}$ source temperature, and $+5500 \mathrm{~V}$ or $-4500 \mathrm{~V}$ spray. Method duration was $4821 \mathrm{~min}$. All target proteins for a given metabolite pool and MS method were analyzed together before 483 switching FIA-MS methods. Between FIA-MS methods, the Shimadzu Nexera HPLC system and SCIEX 484 X500R ESI-QTOF MS where equilibrated for 40 min to the next FIA-MS method. Auto-calibration of positive 485 or negative mode was performed approximately every 45 mins at the beginning of a target protein- 
metabolite pool batch to control detector drift. Non-dialyzed MIDAS metabolite pools were assayed at the beginning, middle, and end of each metabolite pool method batch to control detector sensitivity.

\section{MIDAS data processing and analysis}

490 MIDAS FIA-MS spectra were processed in SCIEX OS 1.6 software using a targeted method to determine metabolite abundances in the protein chamber and metabolite chamber by integrating the mean area under the curve for each extracted ion chromatogram. If necessary, up to one dialysis replicate per pool per protein was removed if processing or autosampling abnormalities were identified. For each dialysis replicate, $\log _{2}$ (fold change) for each metabolite was calculated as the difference between the $\log _{2}$ abundance in the protein chamber and metabolite chamber. $\log _{2}$ (fold change) for metabolite isomers (e.g. L-Leu/L-Ile/L-Allo-Ile) within the same screening pool were collapsed to a single entry prior to further data processing leading to 333 unique metabolite isomer analytes. Using the replicate protein-metabolite $\log _{2}$ (fold change) values as input, a processing method was developed in $R$ (https://github.com/KevinGHicks/MIDAS) to capture and remove extreme outliers and non-specific systematic variation and to determine significant protein-metabolite interactions. Briefly, for each dialysis replicate set, up to one outlier was removed using a $z$-score cutoff of five $(<0.2 \%$ of observations). Technical

502 replicates were then averaged yielding one fold-change summary per protein-metabolite pair. To remove

503 fold-change variation that was not specific to a given protein-metabolite pair, the first three principal 504 components of the total screening dataset were removed on a per metabolite pool basis by subtracting the 505 projection of the first three principal components, creating $\log _{2}$ (corrected fold change). Protein-metabolite $506 \quad z$-scores were determined by comparing the target protein-metabolite $\log _{2}$ (corrected fold change) to a no507 signal model for that metabolite using measures of the central tendency (median) and standard deviation 508 (extrapolated from the inter quartile), which are robust to the signals in the tails of a metabolite's fold-change 509 distribution. Z-scores were false-discovery rate controlled using Storey's q-value (30) and protein510 metabolite interactions with q-values $<0.01$ were considered significant. Since correcting for non-specific 511 binding, and estimating metabolite-specific standard deviation both benefit from the inclusion of additional 512 proteins, MIDAS data from 122 anonymized proteins were analyzed alongside the 38 proteins specifically 513 considered in this study. The complete MIDAS protein-metabolite interaction dataset for mTORC1 514 regulators and enzymes of central carbon metabolism can be found in Data S4.

\section{MIDAS proteins}

517 All presented proteins analyzed by MIDAS were prepared and provided by collaborators (Data S3) using 518 common protein expression and purification techniques. Proteins were received snap frozen on dry ice 519 from outside sources or on wet ice from local sources. Prior to MIDAS screening, protein quality was 520 assessed by $12.5 \%$ SDS-PAGE and concentration was determined by A280 on a NanoDrop One UV-Vis 521 spectrophotometer using the molecular weight and calculated extinction coefficient $\left(\mathrm{M}^{-1} \cdot \mathrm{cm}^{-1}\right)$ of each 522 protein construct. Proteins were screened by MIDAS at the concentrations indicated in Data S3. 
525 MIDAS protein-metabolite interaction data for enzymes of central carbon metabolism were visualized for

526 intra- and inter-pathway relationships using Electrum (v0.0.0; https://github.com/Electrum-app/Electrum),

527 with q-value cutoff $<0.01$, and the 1 -D scaling option enabled.

\section{Differential scanning fluorimetry}

530 Thermal differential scanning fluorimetry (DSF) was performed similar to Niesen et al (31). Briefly, DSF

531 thermal shift assays were developed to assess protein melting point ( $\mathrm{Tm})$ and thermal stability in the

532 presence of putative small molecule ligands: 2-phosphoglycerate (2PG, Sigma-Aldrich 73885),

533 phosphoserine (pSer, Sigma-Aldrich P0878), phosphotyrosine (pTyr, Sigma-Aldrich P9405), phosphate

534 (PO4, Acros Organics 424395000), fumarate (Fum, Sigma-Aldrich 47910), 2-amino-3-

535 phosphonopropionic acid (AP-3, Sigma-Aldrich A4910), ATP (Sigma-Aldrich A2383), ADP (Sigma-Aldrich

536 01905), AMP (Sigma-Aldrich A2252), NAD (Sigma-Aldrich N1636), and palmitoyl-CoA (C16:0-CoA,

537 Avanti 870716). Where indicated, DSF experiments were performed using either the standard SYPRO

538 orange fluorescent system or PROTEOSTAT® Thermal shift stability assay kit (ENZO 51027). A final

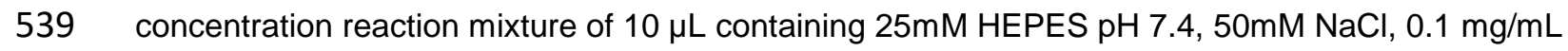

540 (SYPRO system) or $0.75 \mathrm{mg} / \mathrm{mL}$ (PROTEOSTAT system) target protein, 7.5X SYPRO orange (Sigma-

541 Aldrich S5692) or 1x PROTEOSTAT® reagent, and the indicated concentration of putative ligand was

542 arrayed across a MicroAmp ${ }^{\mathrm{TM}}$ optical 384-Well reaction plate (Thermo Scientific 4309849) and sealed

543 with MicroAmp ${ }^{\mathrm{TM}}$ optical adhesive film (Thermo Scientific 4360954). Protein denaturation was measured

544 in sextuplicate technical replicates for SYPRO orange and PROTEOSTAT experiments with an excitation

545 of $470 \mathrm{~nm}$ and emission of $580 \mathrm{~nm}$ on an Applied Biosystems Quantstudio 7 Flex from $25^{\circ} \mathrm{C}$ to $95^{\circ} \mathrm{C}$ at a

546 ramp rate of $0.05^{\circ} \mathrm{C} /$ second. DSF experiments were performed in triplicate. Protein Thermal Shift

547 software 1.4 (Applied Biosystems) was used to interpret and determine protein Tm from the first derivative

548 of the fluorescence emission as a function of temperature $(\mathrm{dF} / \mathrm{dT})$. A change in ligand-induced protein

549 melting point $(\Delta \mathrm{Tm})$ was determined from the difference of the ligand induced $\mathrm{Tm}$ and no-ligand control

550 Tm. Apparent binding affinity (Kdapp) was determined by fitting the specific binding and Hill slope

551 equation to $\Delta T \mathrm{Tm}$ as a function of ligand concentration in GraphPad Prism 9 software.

553 Fumarase competitive inhibition assay

554 The competitive inhibition of human fumarase activity in the presence of 2-amino-3-phosphonopropionic

555 acid (AP-3, Sigma-Aldrich A4910) was fluorometrically assessed using a coupled enzyme assay. Briefly,

556 the rate limiting hydration of fumarate to malate by fumarase provides substrate, malate, for excess

557 malate dehydrogenase to generate oxalacetate and NADH. Fumarase reaction rate was assessed at

558 room temperature in triplicate with a final reaction volume of $100 \mu \mathrm{L}$ composed of $50 \mathrm{mM}$ Tris- $\mathrm{HCl} \mathrm{pH} \mathrm{9.4,}$

$55961 \mu \mathrm{g} / \mathrm{mL}$ human fumarase, excess porcine heart malate dehydrogenase (Sigma-Aldrich 442610-M), 1 
mM NAD (Sigma-Aldrich N1636), and varying concentrations of fumarate (0 - $40 \mathrm{mM}$, Sigma-Aldrich 47910) and AP-3 (0 - 10 mM, Sigma-Aldrich A4910) (Fig. S2). Fumarate and AP-3 were added simultaneously to initiate the reaction. The production of $\mathrm{NADH}$ was quantified fluorometrically in a black, clear bottomed 96-well plate (Sigma-Aldrich CLS3603) on a Biotek Synergy Neo plate reader with $360 \mathrm{~nm}$ excitation and $460 \mathrm{~nm}$ emission over 10 minutes and fumarase reaction rate was determined from the linear range of increasing NADH signal. A Lineweaver-Burke linear regression and non-linear regression competitive inhibition model of human fumarase between fumarate and AP-3 were fit using GraphPad

567 Prism 9 software from triplicate competitive inhibition experiments.

Enolase 2 Activity Assay

570 Human enolase 2 (ENO2) activity was measured in the presence of phosphoserine using a coupled 571 enzyme kinetic assay similar to Satani et al (32). Briefly, enolase converts 2-phosphoglycerate (2PG) to 572 phosphoenolpyruvate (PEP) and water. Substrate, 2PG, was provided near the measured Km. Excess 573 pyruvate kinase (PK) / lactate dehydrogenase (LDH) enzymes from rabbit muscle (Sigma P0294), ADP, 574 NADH were added to solution to ensure that dehydration of 2PG by enolase was the rate-limiting step. 575 Enolase reaction rate was assessed at room temperature in triplicate with a final reaction volume of 100 576 uL composed of $50 \mathrm{mM}$ HEPES pH 7.4, $0.5 \mathrm{mM} \mathrm{MgCl}$, $100 \mathrm{uM} \mathrm{NaCl}, 1.75 \mathrm{mM}$ ADP, 200 uM NADH, $57712.8 \mathrm{U} \mathrm{PK}, 18.4 \mathrm{U} \mathrm{LDH}, 500 \mathrm{ug}$ ng of ENO2, $30 \mathrm{uM}$ 2PG, with varying concentrations of phosphoserine 578 (pSer). 2PG was used to initiate the coupled enzyme reaction, and the conversion of NADH to NAD by 579 LDH was quantified fluorometrically in a black, clear bottomed 96-well plate (Sigma-Aldrich CLS3603) on

580 a Biotek Synergy Neo plate reader with $360 \mathrm{~nm}$ excitation and $460 \mathrm{~nm}$ emission over 10 minutes.

581 Enolase reaction rate was determined for the linear range of decreasing NADH signal. IC50 was determined using a sigmoidal, 4PL non-linear regression in Prism 9 from triplicate experiments.

\section{Enolase X-ray crystallography}

585 Crystals of Human Enolase 2 in complex with the phosphoserine ligand were prepared via hanging drop 586 vapor diffusion at $20^{\circ} \mathrm{C}$. $9 \mathrm{mg} / \mathrm{ml}$ - Human Enolase 2 protein solution with $2 \mathrm{mM}$ phosphoserine was pre587 incubated on ice for 10 min prior to being mixed in 1:1 ratio (protein:reservoir solution) with $100 \mathrm{mM}$ Bis 588 Tris, $200 \mathrm{mM}$ ammonium acetate and 21\% (w/v) PEG 3350 at pH 6.5. Orthorhombic crystals grew within 5893 days and were subsequently cryoprotected with $100 \mathrm{mM}$ Bis Tris, $200 \mathrm{mM}$ ammonium acetate, 32\% 590 (w/v) PEG 3350 and 2 mM phosphoserine. X-ray diffraction data were collected at the Advanced Photon 591 Source, synchrotron beamline 22-ID, equipped with Si(III) monochromator and EIGER CCD detector. The 592 diffraction data was processed and integrated using iMOSFLM (33). POINTLESS (34) was used to 593 identify the bravais lattice and space group and AIMLESS (35) was used for scaling. The phase 594 information was obtained by molecular replacement using PHASER (36) with a homodimer of Human 595 Enolase 2 (PDB 4ZCW) as the search model. Iterative cycles of manual model building and refinement 
were performed within Phenix (37) and COOT (38) software. Diffraction data and refinement statistics are summarized in Table S1.

\section{Fumarase X-ray crystallography}

600 Human fumarase ( $\mathrm{HsFH}$ ) was produced and purified as previously described (39). The co-crystallization 601 experiments were carried out by using the sitting drop method. Protein solution $(4 \mathrm{mg} / \mathrm{mL}$ in $50 \mathrm{mM}$ Tris$602 \mathrm{HCl}$ (Sigma-Aldrich), pH 8.5, $150 \mathrm{mM} \mathrm{KCl}$ (J.T.Baker) was incubated with $20 \mathrm{mM}$ of D-2-amino-3603 phosphono-propionic acid (AP-3). $2 \mu \mathrm{L}$ of protein solution was mixed with $2 \mu \mathrm{L}$ of reservoir solution, and 604 allowed to equilibrate against $500 \mu \mathrm{l}$ of reservoir solution at $21^{\circ} \mathrm{C}$. Crystals occur over the course of 3 605 days in drops where the reservoir contained $100 \mathrm{mM}$ Hepes pH 7.5 (Sigma-Aldrich), 1\% v/v 2606 methylpentanediol (MPD) (Sigma-Aldrich) and 18\% (w/v) PEG $10 \mathrm{~K}$ (Sigma-Aldrich) and 25\% (v/v) 607 glycerol. Prior to data collection, HsFH crystals were soaked in a cryoprotectant solution (100 mM Hepes $608 \mathrm{pH} 7.5,1 \% \mathrm{v} / \mathrm{v}$ 2-methylpentanediol (MPD), and 18\% m/v PEG $10 \mathrm{~K}, 25 \% \mathrm{v} / \mathrm{v}$ glycerol (Labsynth), 609 harvested with cryo loops, and flash-cooled in liquid nitrogen. The data set was collected at $100 \mathrm{~K}$ on a 610 synchrotron facility (MANACA beamline - SIRIUS, Brazil) using a PILATUS 2M detector (Dectris). 3600 611 frames with an oscillation step of $0.1^{\circ}$ were collected using an exposure time of $0.1 \mathrm{~s}$ per image with a 612 crystal-to-detector distance of $120.05 \mathrm{~mm}$. The images of X-ray diffraction were processed with XDS (40)

613 package, and the structure of $\mathrm{HsFH}$ was solved by molecular replacement implemented in Molrep (41)

614 program, and using the human fumarase structure (PDB ID: 5UPP) (39) as a template. The structure was 615 refined with Refmac5 (42) intercepted with manual map inspection and model building using Coot (38).

616 The quality of the model was regularly checked using MolProbity (43). Diffraction data and refinement

617 statistics are summarized in Table S2. The refined atomic coordinates and structure factors were

618 deposited in the PDB with the accession code 7LUB.

\section{Lactate dehydrogenase activity assay}

621 Human lactate dehydrogenase A (LDHA) and lactate dehydrogenase B (LDHB) activity were assessed in 622 the presence of putative nucleotide and fatty acyl-CoA derivatives using a standard NADH fluorometric 623 assay. Briefly, lactate dehydrogenase reversibly converts lactate and NAD to pyruvate and NADH. With 624 the exception of the competitive inhibition assay, LDHA and LDHB activity assays were operated near 625 their measured substrate and cofactor $\mathrm{Km}$. Lactate dehydrogenase reaction rate was assessed at room 626 temperature in triplicate with a final reaction volume of $100 \mu \mathrm{L}$ composed of $75 \mathrm{mM} \mathrm{Tris} \mathrm{pH} 7.4,67.2$ $627 \mathrm{ng} / \mathrm{ml}$ LDHA or $75 \mathrm{ng} / \mathrm{mL}$ LDHB, $6.5 \mathrm{mM}$ Lactate (Sigma-Aldrich L6402) and $200 \mu \mathrm{M}$ NAD (Sigma-Aldrich $628 \mathrm{~N} 1636)$ for LDHA and $1 \mathrm{mM}$ lactate and $1.25 \mathrm{mM}$ NAD for LDHB, with varying concentrations of putative 629 ligand, as indicated: ATP (Sigma-Aldrich A2383), ADP (Sigma-Aldrich 01905), AMP (Sigma-Aldrich 630 A2252), CoA (Avanti 870701), C2:0-CoA (Avanti 870702), C4:0-CoA (Avanti 870704), C8:0-CoA (Avanti 631 870708), C12:0-CoA (Avanti 870712), C16:0-CoA (Avanti 870716), C18:1-CoA (Avanti 870719), and 632 C20:0-CoA (Avanti 870720). For competitive inhibition assay, the concentrations of lactate or NAD were 
633 varied, accordingly. In all circumstances, NAD was used to initiate the lactate dehydrogenase reaction.

634 The production of NADH was quantified fluorometrically in a black, clear bottomed 96-well plate (Sigma-

635 Aldrich CLS3603) on a Biotek Synergy Neo plate reader with $360 \mathrm{~nm}$ excitation and $460 \mathrm{~nm}$ emission

636 over 10 minutes and lactate dehydrogenase reaction rate was determined from the linear range of

637 increasing NADH signal. IC S0 $_{50}$ were determined using a sigmoidal, 4PL non-linear regression in Prism 9

638 from triplicate experiments. Non-linear regression competitive or non-competitive inhibition modeling of

639 LDHA between lactate or NAD and ATP or palmitoyl-CoA were fit using GraphPad Prism 9 software from

640 triplicate experiments.

642 Palmitoyl-CoA-Agarose pull-down assay

643 LDHA and LDHB interaction with palmitoyl-CoA was assessed using a pull-down, competitive elution

644 assay. Briefly, $30 \mu \mathrm{L}$ per pull-down of palmitoyl-CoA conjugated agarose beads (Sigma-Aldrich 5297)

645 were buffer exchanged into pull-down buffer (75mM Tris $\mathrm{HCl} \mathrm{pH} \mathrm{7.4).} \mathrm{In} \mathrm{a} \mathrm{final} \mathrm{volume} \mathrm{of} 300 \mu \mathrm{L}, 0.2$

$646 \mathrm{mg} / \mathrm{mL}$ of LDHA or LDHB protein were combined with buffer exchanged palmitoyl-CoA agarose beads, a

647 loading control was saved, and the mixture was incubated overnight at $4^{\circ} \mathrm{C}$ with gentle agitation. Post-

648 incubation, pull-down reactions were washed $5 \mathrm{x}$ in $100 \mu \mathrm{L}$ of pull-down buffer and the final wash was

649 saved for analysis. Following the fifth wash, $100 \mu \mathrm{M}$ of acetyl-CoA or palmitoyl-CoA or equivalent volume

650 of pull-down buffer were added to the reactions and incubated overnight at $4^{\circ} \mathrm{C}$. In the morning, the pull-

651 down reactions were centrifuged to pellet beads, the supernatant was collected and concentrated as the

652 eluate fraction, the beads were collected as the bound fraction, and all samples were boiled for 5 minutes

653 in 4x Laemmli sample buffer and analyzed by SDS-PAGE for presence of LDHA or LDHB.

Tissue culture

656 H9c2 myoblastic cell line (ATCC CRL-1446) was purchased from ATCC and routinely maintained in

657 DMEM media supplemented with 10\% FBS and 1\% PenStrep in $5 \%$ CO2 and $37^{\circ} \mathrm{C}$.

Ldha and Ldhb mutant cell lines

660 Ldha and Ldhb knock out H9c2 cell lines were generated using CRISPR-Cas9 to excise the first coding

661 exon of each gene. Single guide modified synthetic sgRNAs were obtained from Synthego and Hifi-Cas9

662 was obtained from IDT (cat\# 1081060). Pairs of ribonucleoprotein (RNP) complexes targeting upstream

663 and downstream of the first coding exon for each gene were co-electroporated using a Lonza 4D

664 Nucleofector system (https://knowledge.lonza.com/cell?id=1016\&search=H9c2). The N20 sgRNA target

665 sequences used were GAGTGCAACGCTCAACGCCA and TCCACAGGCTTGTGACATAA for Ldha and

666 TCCATGCATGTAAAGCACAT and AAGACAGCACAACTCTATAG for Ldhb. Off-targets for these

667 sgRNAs were screened using CasOT (44). Nucleofected cells were plated as single clones and clones

668 were screened for the expected genomic deletion and presence of the WT allele using PCR and these

669 results were confirmed using Western blotting. 
Cell lysate and western blotting

672 Harvested cells were washed with PBS and lysed in RIPA buffer (50 mM Tris, $150 \mathrm{mM} \mathrm{NaCl}, 0.1 \%$ SDS,

$6730.5 \%$ sodium deoxycholate, $1 \%$ NP-40) supplemented with protease and phosphatase inhibitors. Protein

674 concentration was quantified with the Pierce BCA Protein Assay Kit. Samples were mixed with 4x sample

675 loading buffer and incubated for $5 \mathrm{~min}$ at $95^{\circ} \mathrm{C}$. $30 \mu \mathrm{g}$ of total protein lysate was resolved on SDS

676 polyacrylamide gel according to standard procedure at $20 \mathrm{~mA}$ per gel and blotted onto a nitrocellulose

677 membrane $0.45 \mu \mathrm{m}$ (GE Healthcare) via Mini Trans-blot module (Bio-Rad) at a constant voltage (100 V) for

$6782 \mathrm{~h}$. After blocking with 5\% non-fat milk (Serva)/Tris-buffered saline with $0.05 \%$ Tween 20 (TBS-T) for $1 \mathrm{~h}$,

679 the membrane was incubated overnight in 5\% bovine serum albumin (Sigma-Aldrich), TBS-T with primary 680 antibody against LDHA (Cell Signaling Technology 2012S, 1:1000), LDHB (Abcam ab240482, $0.1 \mathrm{~g} / \mathrm{ml}$ ), 681 and GAPDH (Cell Signaling Technology 97166, 1:1000). Next day, the membrane was washed with TBS-

$682 \mathrm{~T}$ and incubated with corresponding fluorophore-conjugated secondary antibody (Rockland 683 Immunochemical RL611-145-002, 1:10000) in 1\% non-fat milk/TBS-T for $1 \mathrm{~h}$. The membrane was then 684 washed again with TBS-T and fluorescence was assessed with Odyssey CLx imaging system (LI-COR 685 Biosciences).

Metabolite extraction

688 The procedures for metabolite extraction from cultured cells are described in previous studies (45-47). 689 Briefly, adherent cells were grown in $10 \mathrm{~cm}$ plates in biological triplicate to $80 \%$ confluence, medium was 690 rapidly aspirated and cells were washed with cold $0.9 \% \mathrm{NaCl}$ TC grade (Sigma-Aldrich S8776-100ML) on 691 ice. $3 \mathrm{~mL}$ of extraction solvent, 80\% (v/v) LC/MS grade methanol/water (Fisher Scientific W6-1, A456-1) 692 cooled to $-80^{\circ} \mathrm{C}$, was added to each well, and the dishes were transferred to $-80^{\circ} \mathrm{C}$ for $15 \mathrm{~min}$. Cells were 693 then scraped into the extraction solvent on dry ice. Additionally, $300 \mathrm{~mL}$ of media was collected and 694 processed from each sample pre and post experiment. All metabolite extracts were centrifuged at 20,000 $695 \times \mathrm{g}$ at $4^{\circ} \mathrm{C}$ for $10 \mathrm{~min}$. Each sample was transferred to a new $1.5 \mathrm{~mL}$ tube. Finally, the solvent in each sample was evaporated in a Speed Vacuum, and stored at $-80^{\circ} \mathrm{C}$ until they were run on the mass spectrometer.

\section{[U-13C6]-Glucose and [U-13C3]-Lactate labeling with or without palmitate}

700 Cells were grown to $80 \%$ confluence in $10 \mathrm{~cm}$ plates with standard culture medium at which point $10 \mu \mathrm{M}$ of 701 the MPC inhibitor UK5099 (Sigma-Aldrich PZ0160-5MG) was added for 48 hours to facilitate lactate 702 production. Cells were subsequently washed with sterile PBS and either free BSA or BSA conjugated to 703 palmitate (Caymen Chemical 29558) was added to culture media containing either [U-13C6]-L-glucose, or 704 [U-13C3]-L-lactate (Cambridge Isotope Laboratories CLM-1396, CLM-1579-PK), supplemented with 705 dialyzed Fetal Bovine Serum (Thermo Scientific A3882001) and incubated for 4 hours. Metabolites were 706 extracted as described above. Data was corrected for naturally occurring $13 \mathrm{C}$ isotope abundance before 
707 analysis as described in Buescher et al (48). All data expressed as mean \pm SD unless otherwise indicated.

708 Student's $t$ test was used for 2 group comparison. One-Way ANOVA and Sidak's comparisons were used 709 for multigroup comparison. $p<0.05$ were considered statistically significant. Statistical analyses and 710 graphics were carried out with GraphPad Prism 9 software.

Metabolomic analysis

713 The levels of metabolites in the H9c2 cells were measured by gas chromatography-mass spectroscopy 714 (GC-MS) analysis. All GC-MS analysis was performed with a Waters GCT Premier mass spectrometer 715 fitted with an Agilent 6890 gas chromatograph and a Gerstel MPS2 autosampler. Dried samples were 716 suspended in $40 \mu \mathrm{L}$ of a $40 \mathrm{mg} / \mathrm{mL}$ O-methoxylamine hydrochloride (MOX) in pyridine and incubated for 1 $717 \mathrm{~h}$ at $30^{\circ} \mathrm{C} .10 \mu \mathrm{L}$ of $\mathrm{N}$-methyl-N-trimethylsilyltrifluoracetamide (MSTFA) was added automatically via the 718 autosampler and incubated for $60 \mathrm{~min}$ at $37^{\circ} \mathrm{C}$ with shaking. After incubation $3 \mu \mathrm{L}$ of a fatty acid methyl 719 ester standard solution was added via the autosampler. Then $1 \mu \mathrm{L}$ of the prepared sample was injected to 720 the gas chromatograph inlet in the split mode with the inlet temperature held at $250^{\circ} \mathrm{C}$. A $10: 1$ split ratio 721 was used for analysis. The gas chromatograph had an initial temperature of $95^{\circ} \mathrm{C}$ for one minute followed 722 by a $40^{\circ} \mathrm{C} / \mathrm{min}$ ramp to $110^{\circ} \mathrm{C}$ and a hold time of $2 \mathrm{~min}$. This was followed by a second $5^{\circ} \mathrm{C} / \mathrm{min}$ ramp to $723250^{\circ} \mathrm{C}$, a third ramp to $350^{\circ} \mathrm{C}$, then a final hold time of $3 \mathrm{~min}$. A $30 \mathrm{~m}$ Phenomex ZB5-5 MSi column with a $7245 \mathrm{~m}$ long guard column was employed for chromatographic separation. Helium was used as the carrier gas 725 at $1 \mathrm{~mL} / \mathrm{min}$. Data was extracted from each chromatogram as area under the curve for individual 726 metabolites. Each sample was first normalized to the added standard d4-succinate to account for extraction 727 efficiency followed by normalization to cell number. Due to this being a broad scope metabolomics analysis, 728 no normalization for ionization efficiency or concentration standards was performed. 
EI 1 bioRxiv preprint doi: https://doi.org/10.1101/2021.08.28.458030; this version posted August 28, 2021. The copyright holder for this preprint which was not certified by peer review) is the author/funder, who has granted bioRxiv a license to display the preprint in perpetuity. It is made available under aCC-BY-NC-ND 4.0 International license.

A

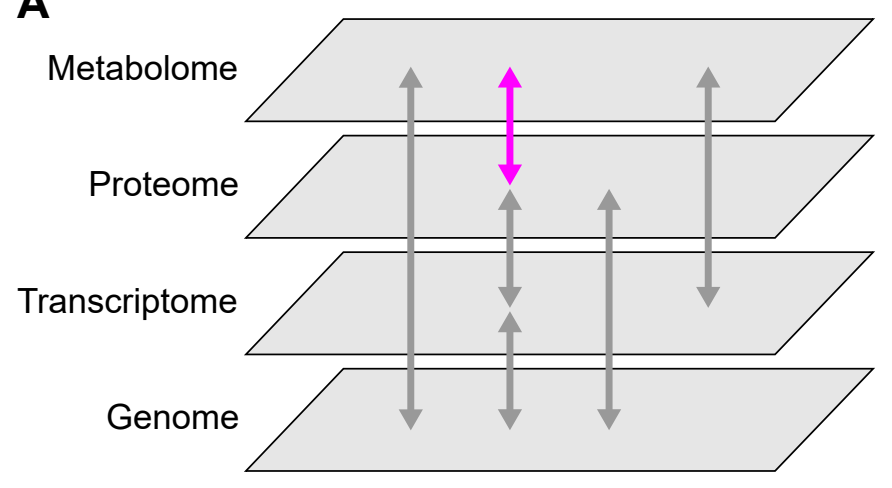

C

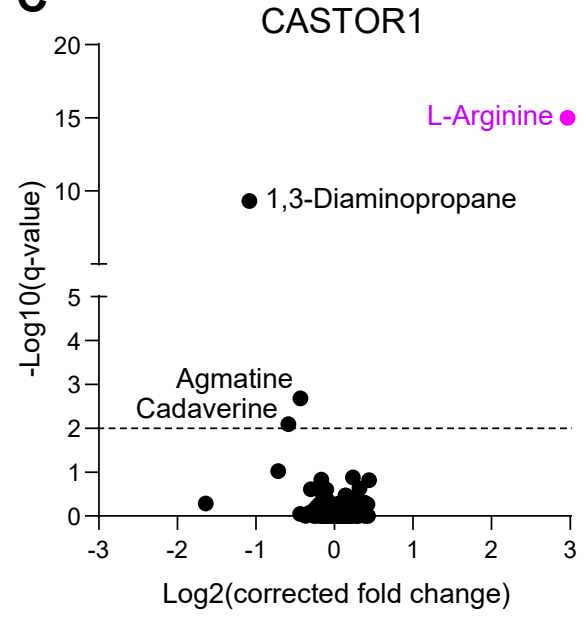

D
B
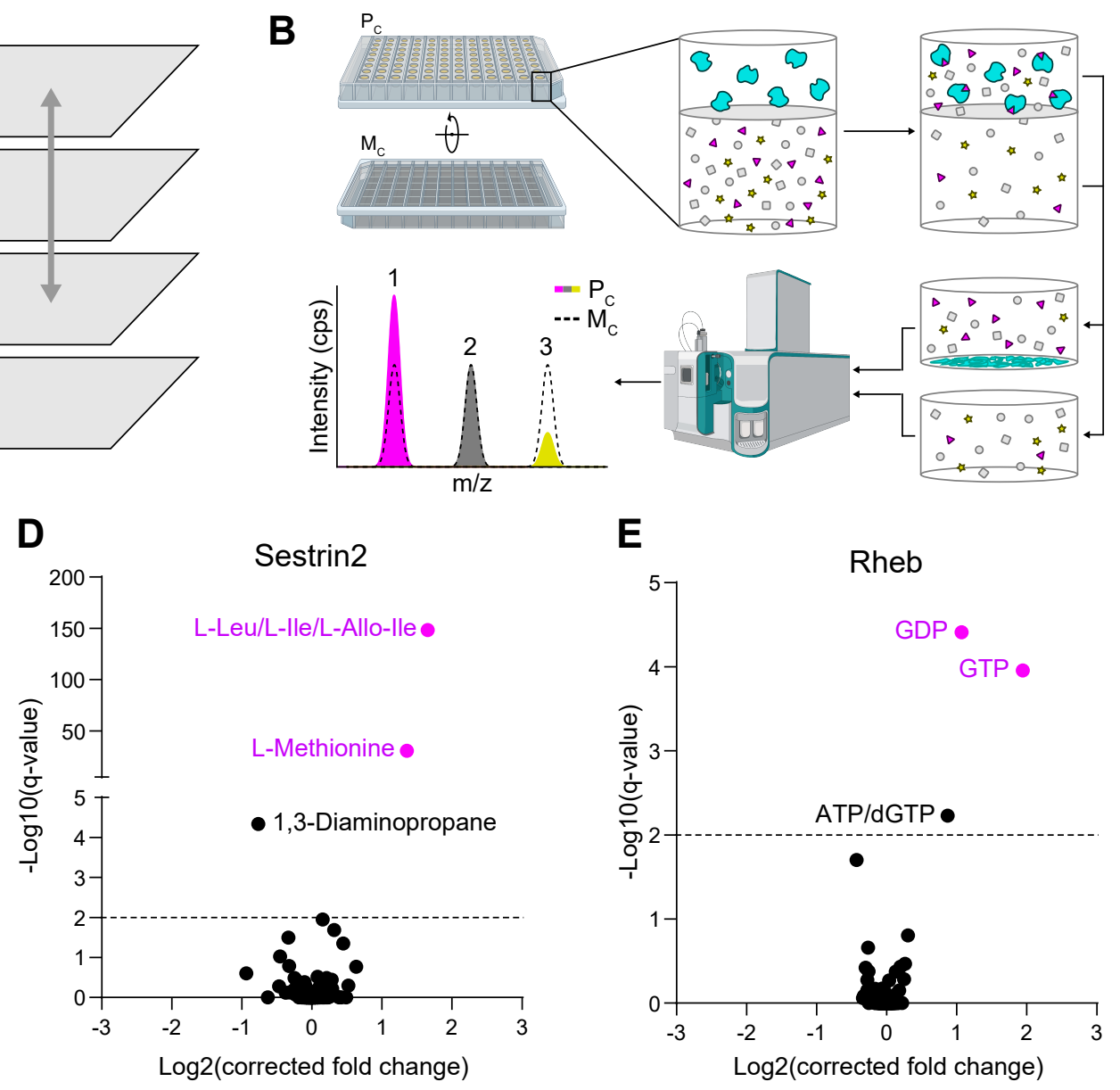

E

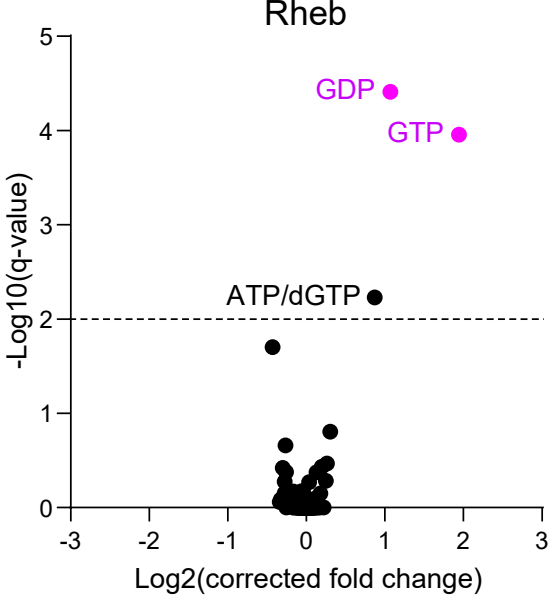




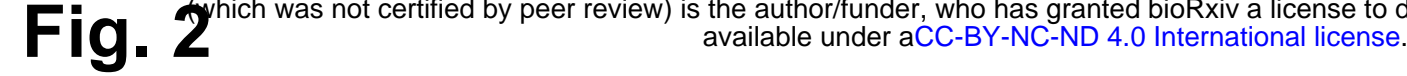

A

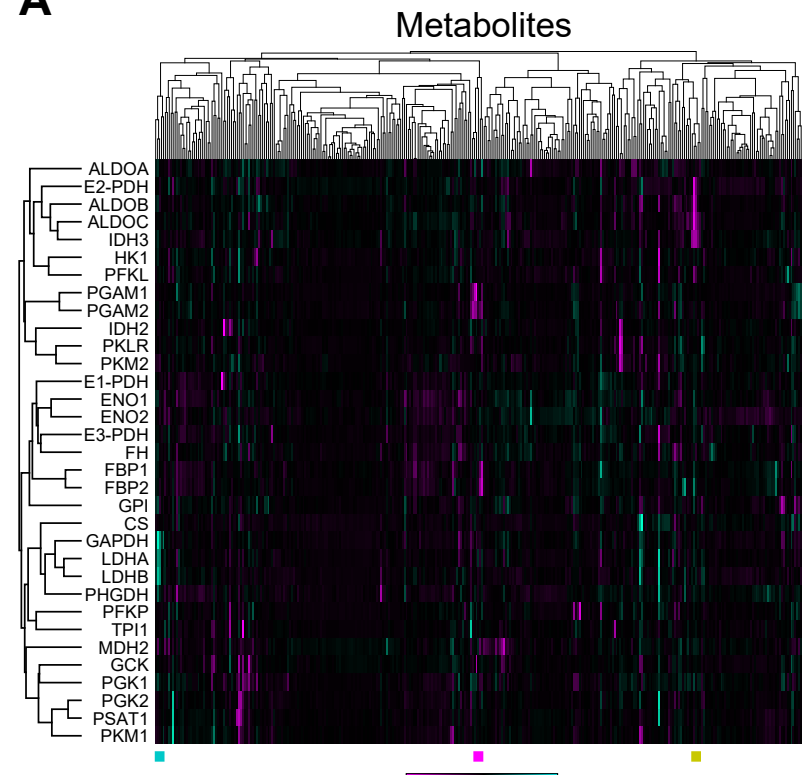

B

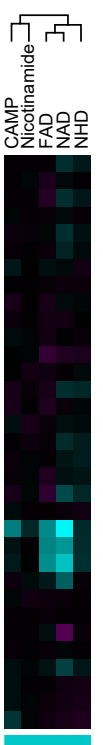

C D ता म म

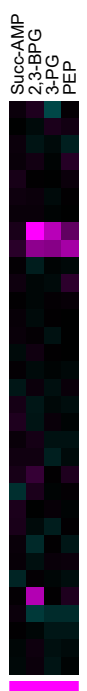

E

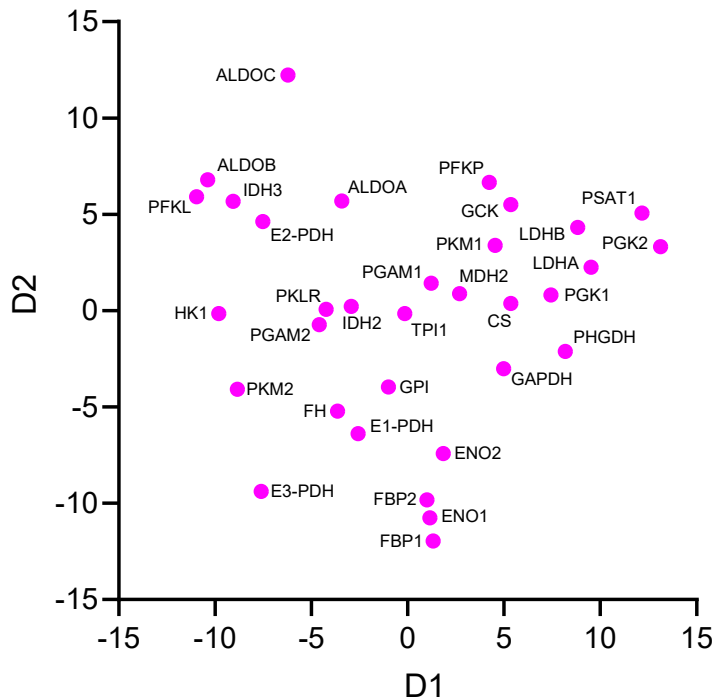

F

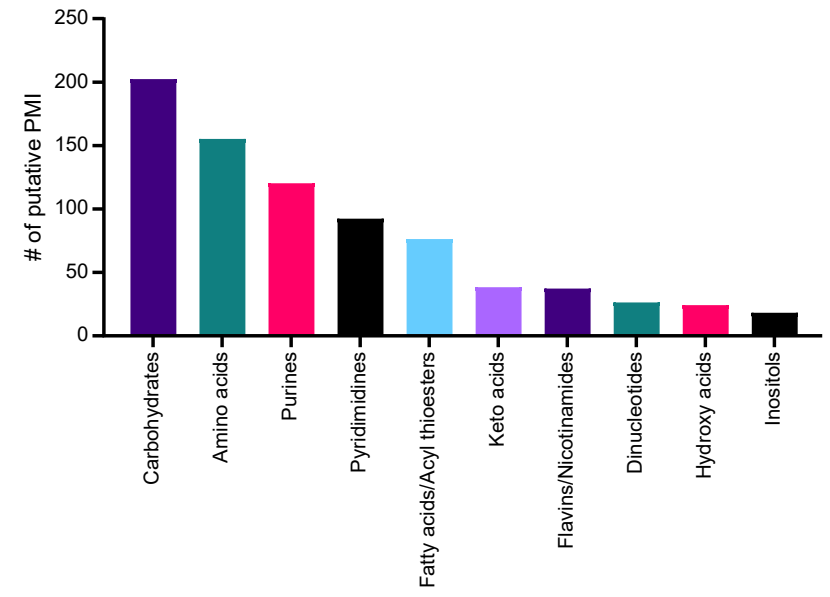

H

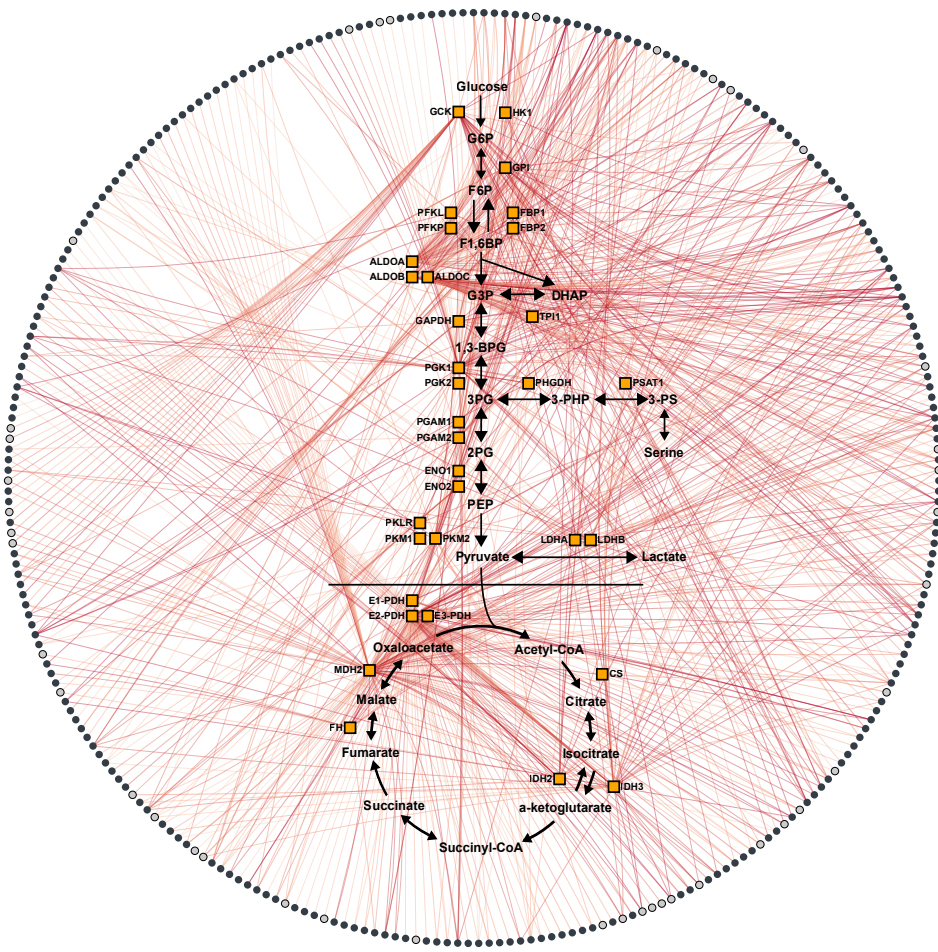

G

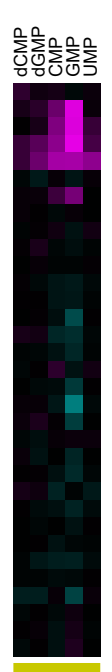

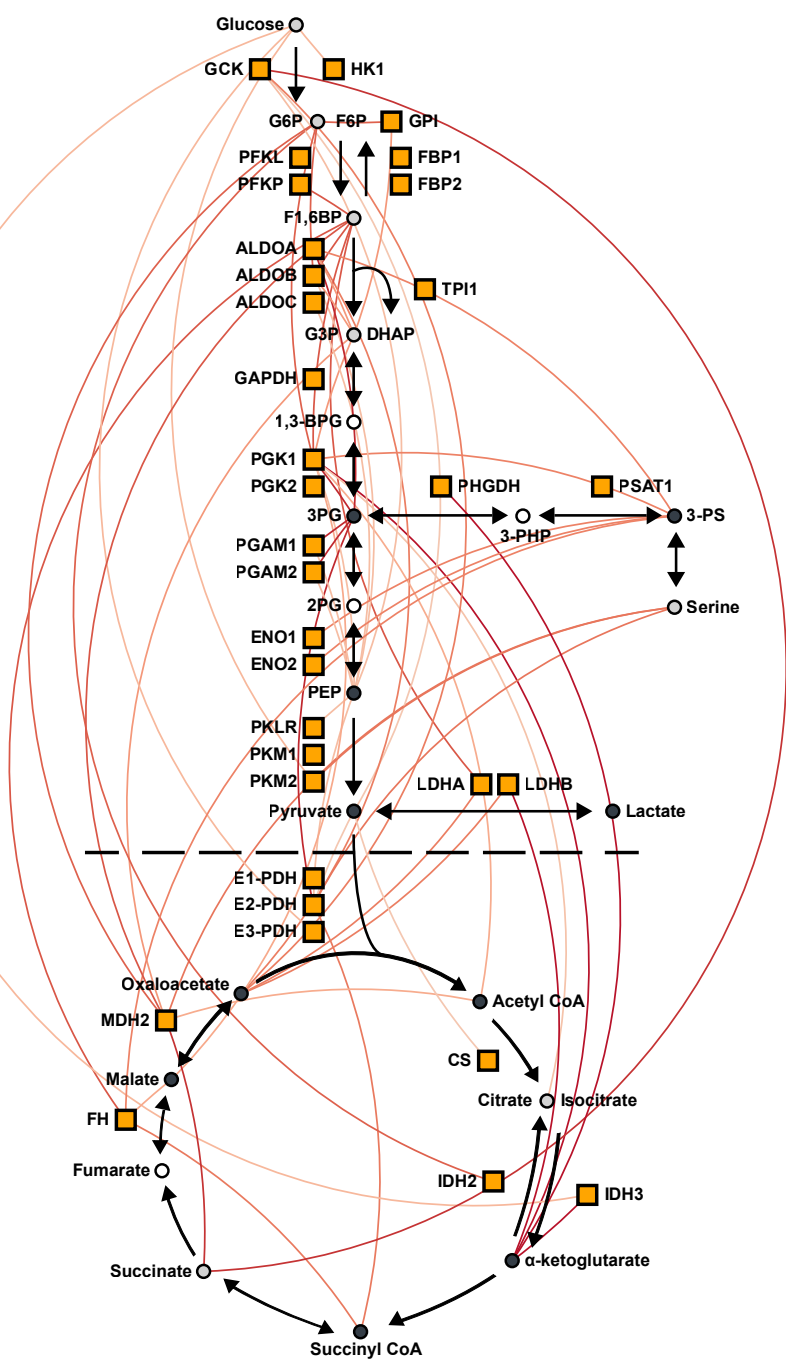


2ioRxiv preprint doi: https://doi.org/10.1101/2021.08.28.458030; this version posted August 28, 2021. The copyright holder for this preprint

19. Which was not certified by peer review) is the author/funder, who has granted bioRxiv a license to display the preprint in perpetuity. It is made available under aCC-BY-NC-ND 4.0 International license.

A ENO1 ENO2

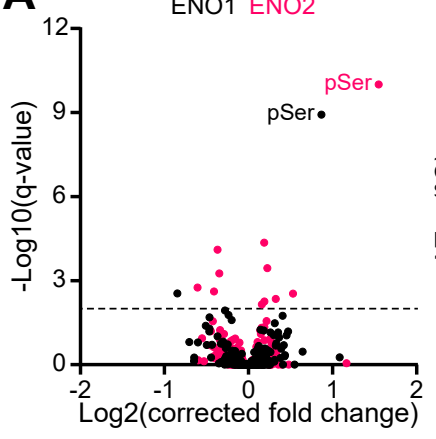

E

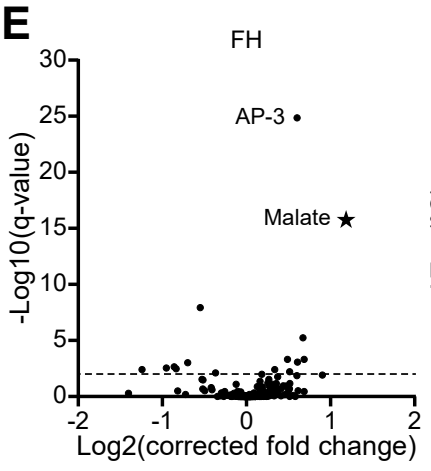

I
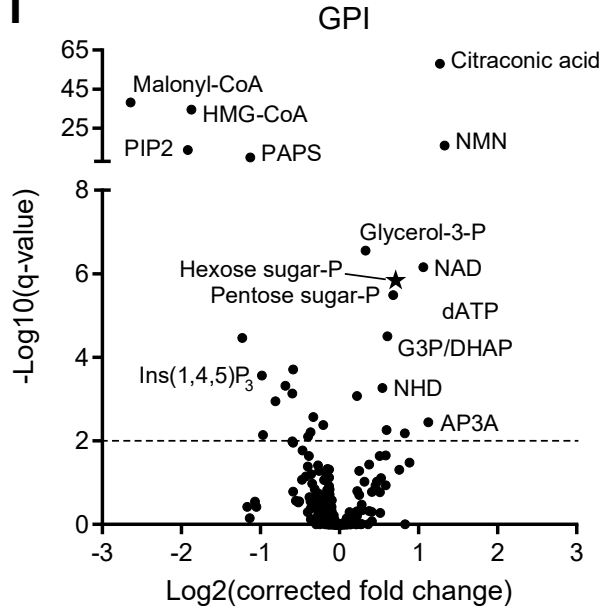

$\mathbf{L}$

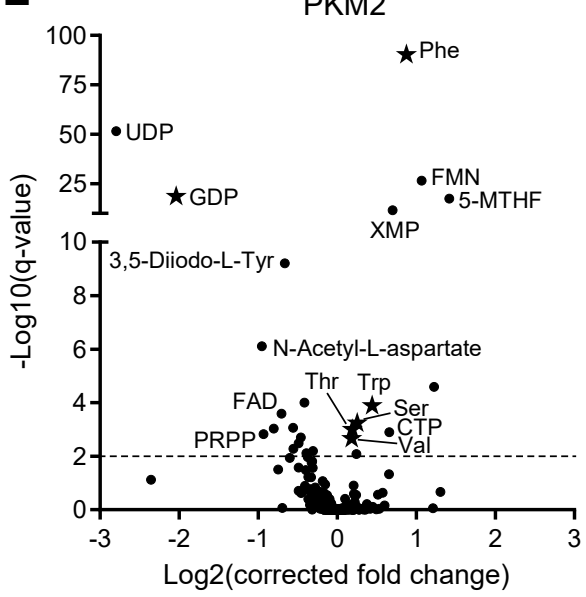

C

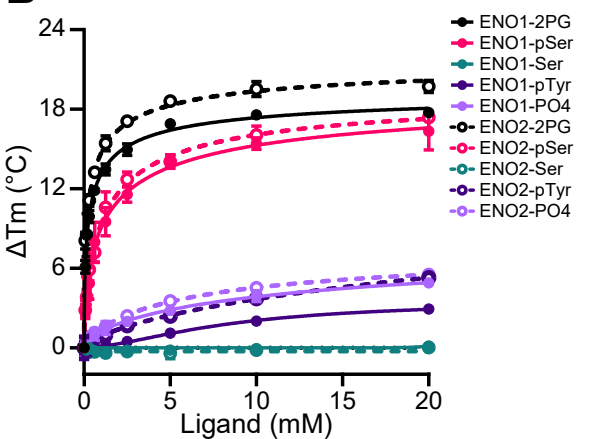

$\mathbf{F}$

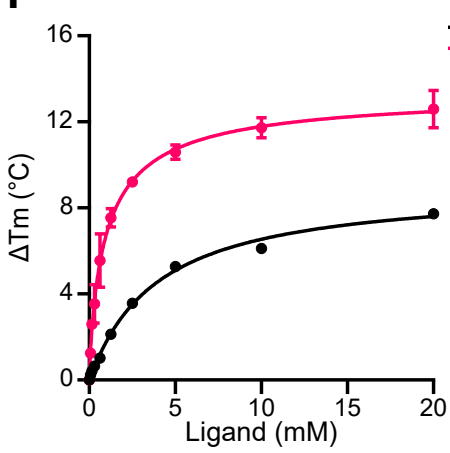

G

$\underset{* \text { FH-AP-3 }}{* \text { FHe }}$

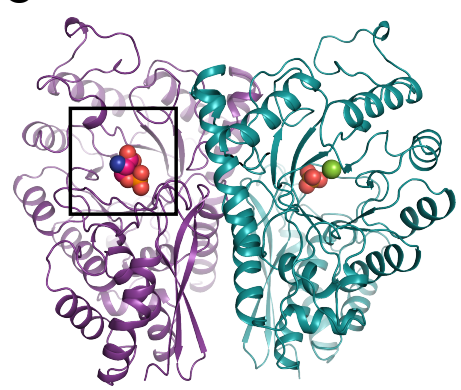

D
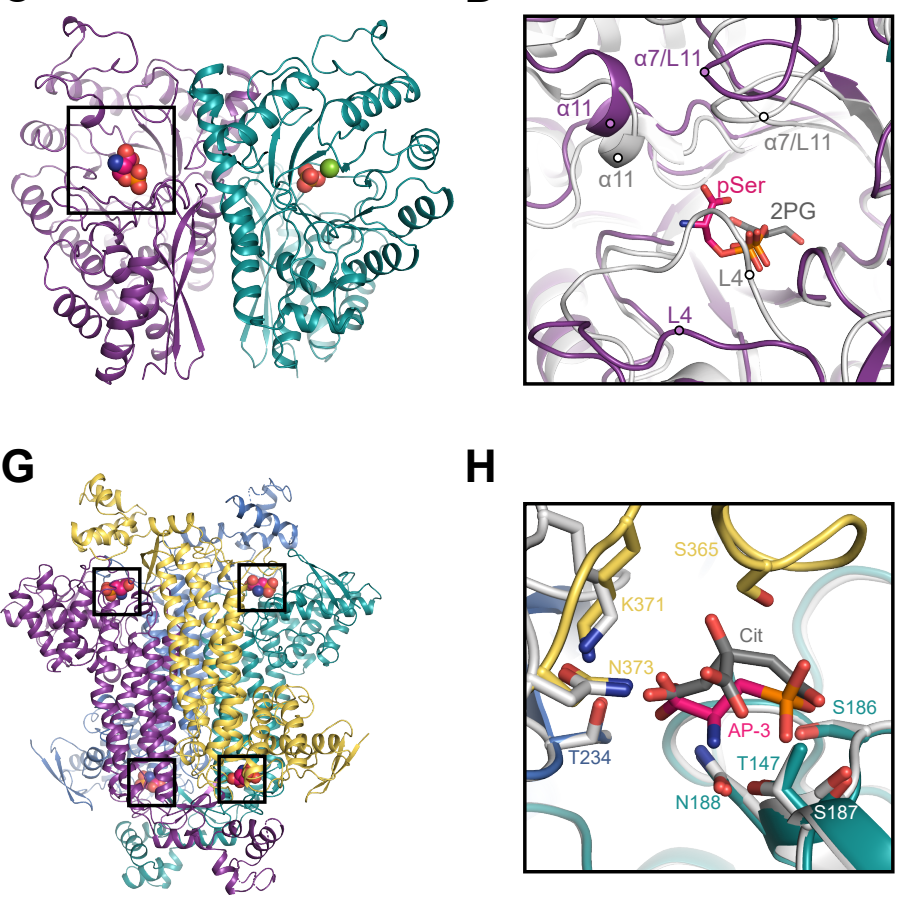

PFKP

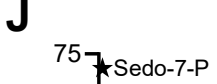

K

${ }_{100}$

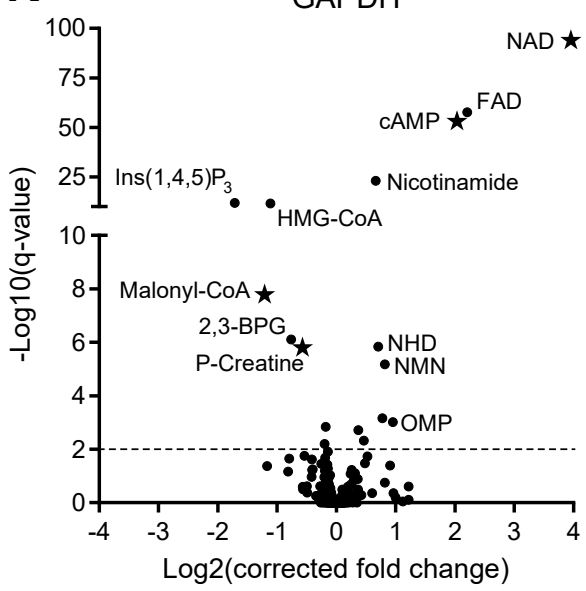

$\mathbf{N}$

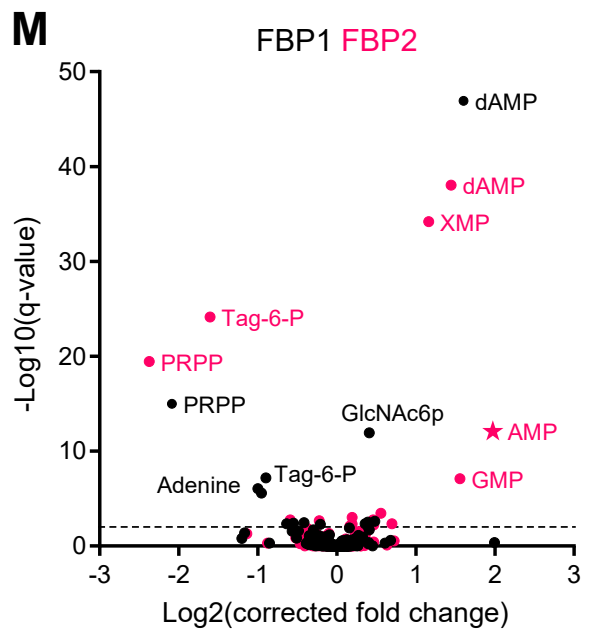

H

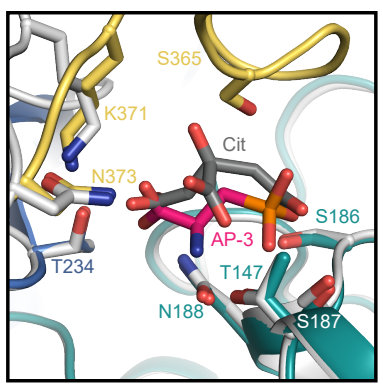

GAPDH
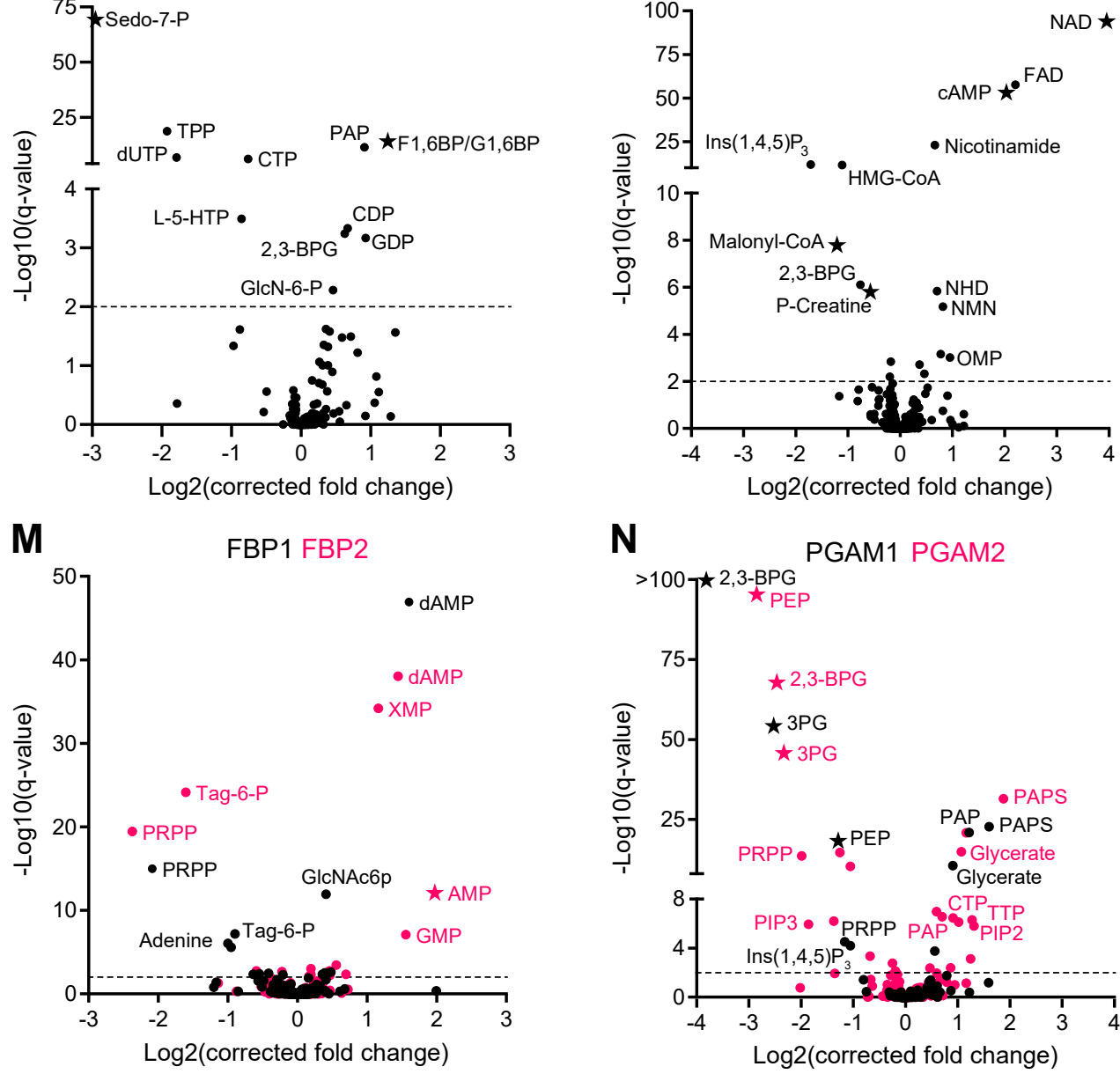
EioRxiv preprint doi: https://doi.org/10.1101/2021.08.28.458030; this version posted August 28, 2021. The copyright holder for this preprint 19. 4hich was not certified by peer review) is the author/funder, who has granted bioRxiv a license to display the preprint in perpetuity. It is made
available under aCC-BY-NC-ND 4.0 International license.

A

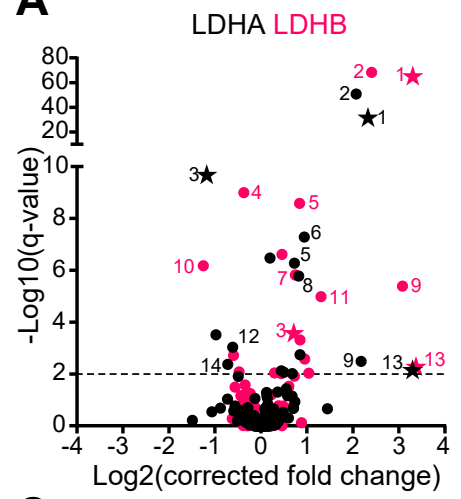

C

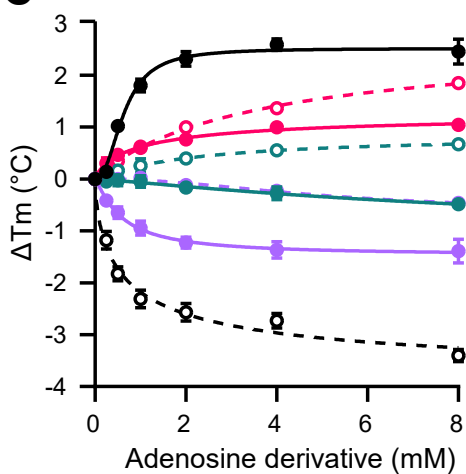

E

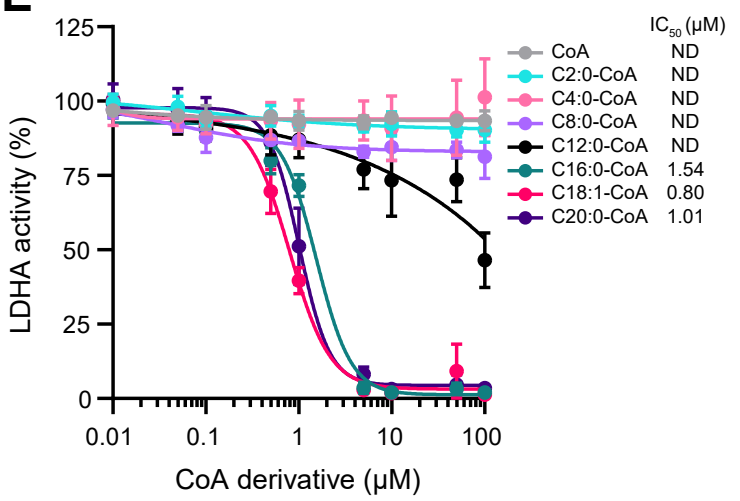

G

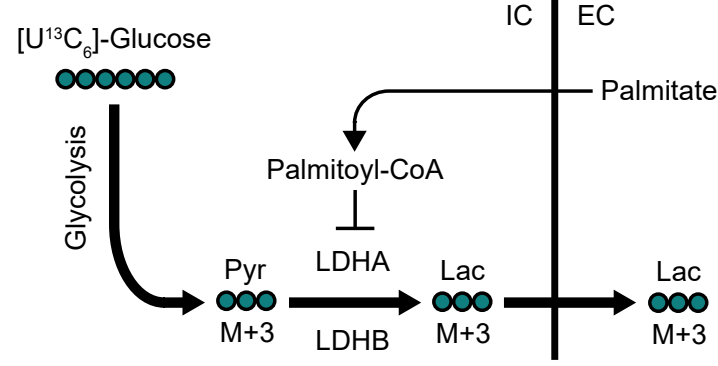

I

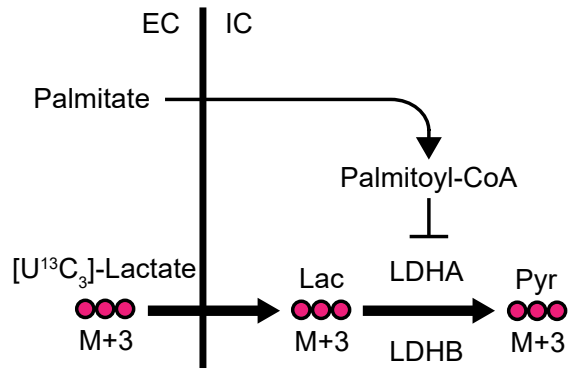

B

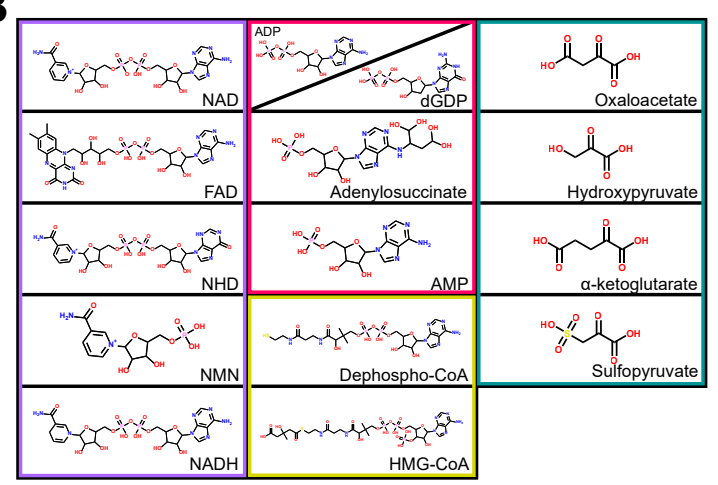

D

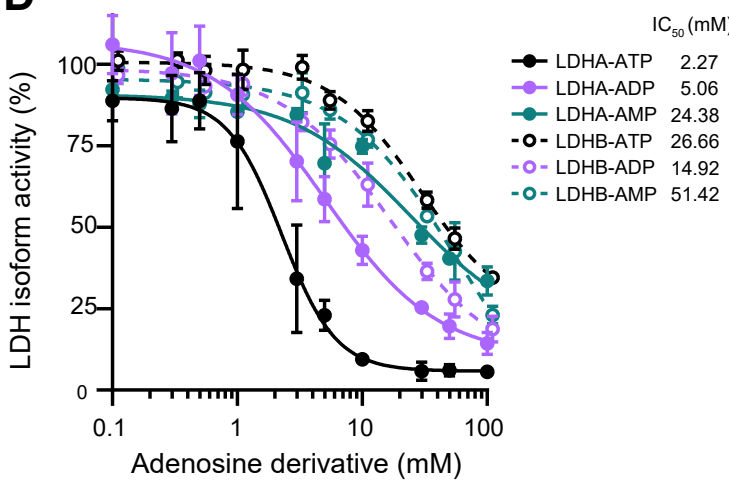

F

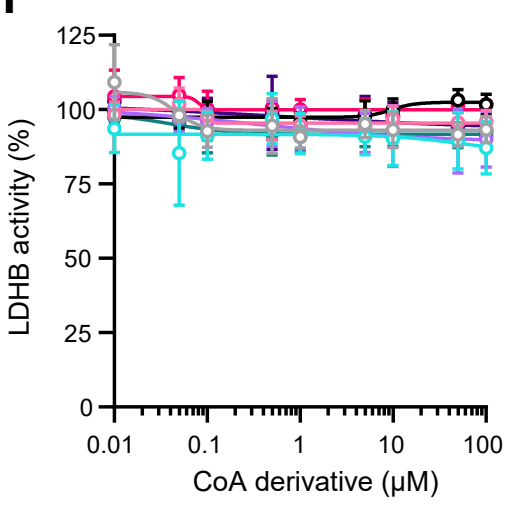

H
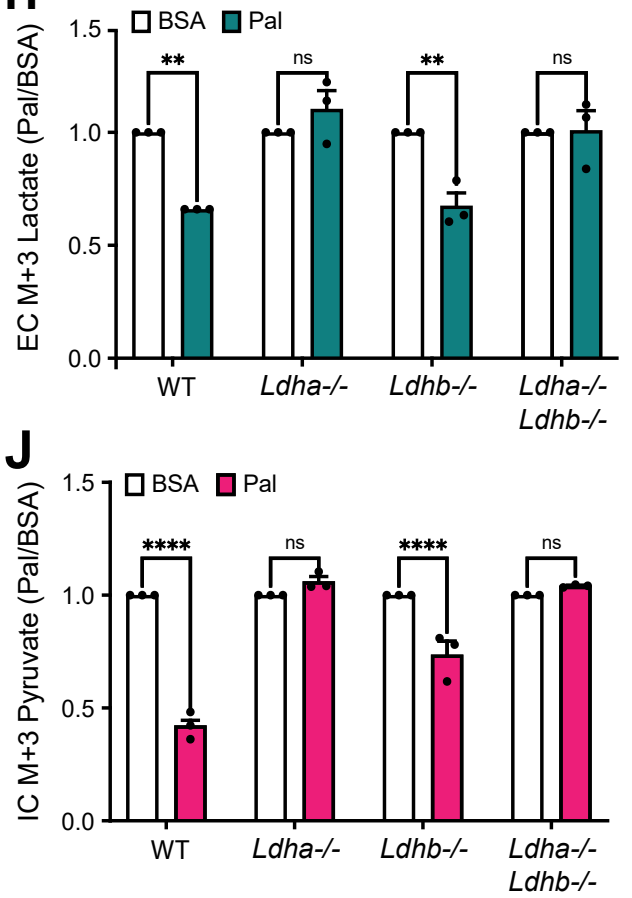
EI Pit Rxiv preprint doi: https://doi.org/10.1101/2021.08.28.458030; this version posted August 28, 2021. The copyright holder for this preprint

19. Whilh was not certified by peer review) is the author/funder, who has granted bioRxiv a license to display the preprint in perpetuity. It is made available under aCC-BY-NC-ND 4.0 International license.

A

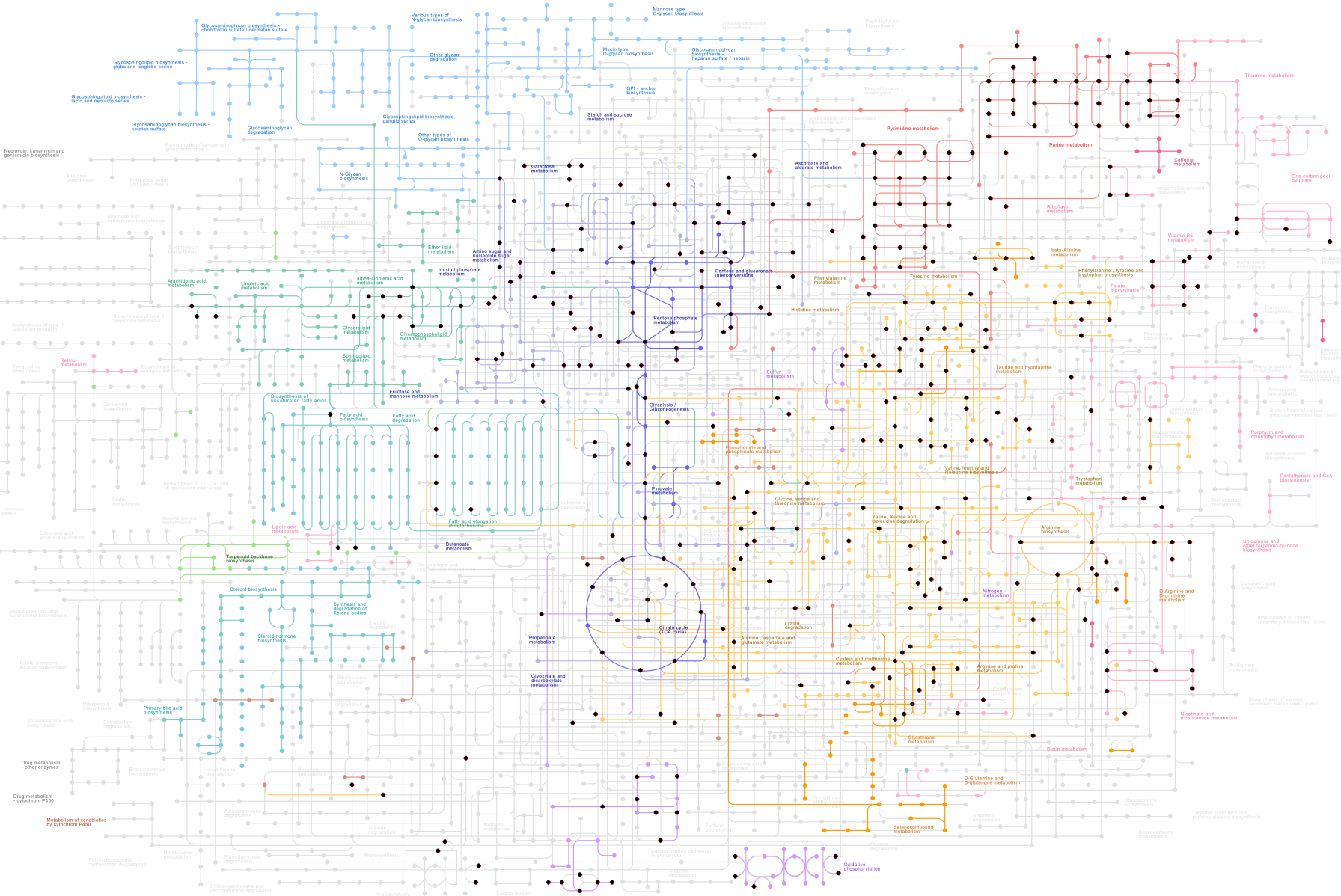

B
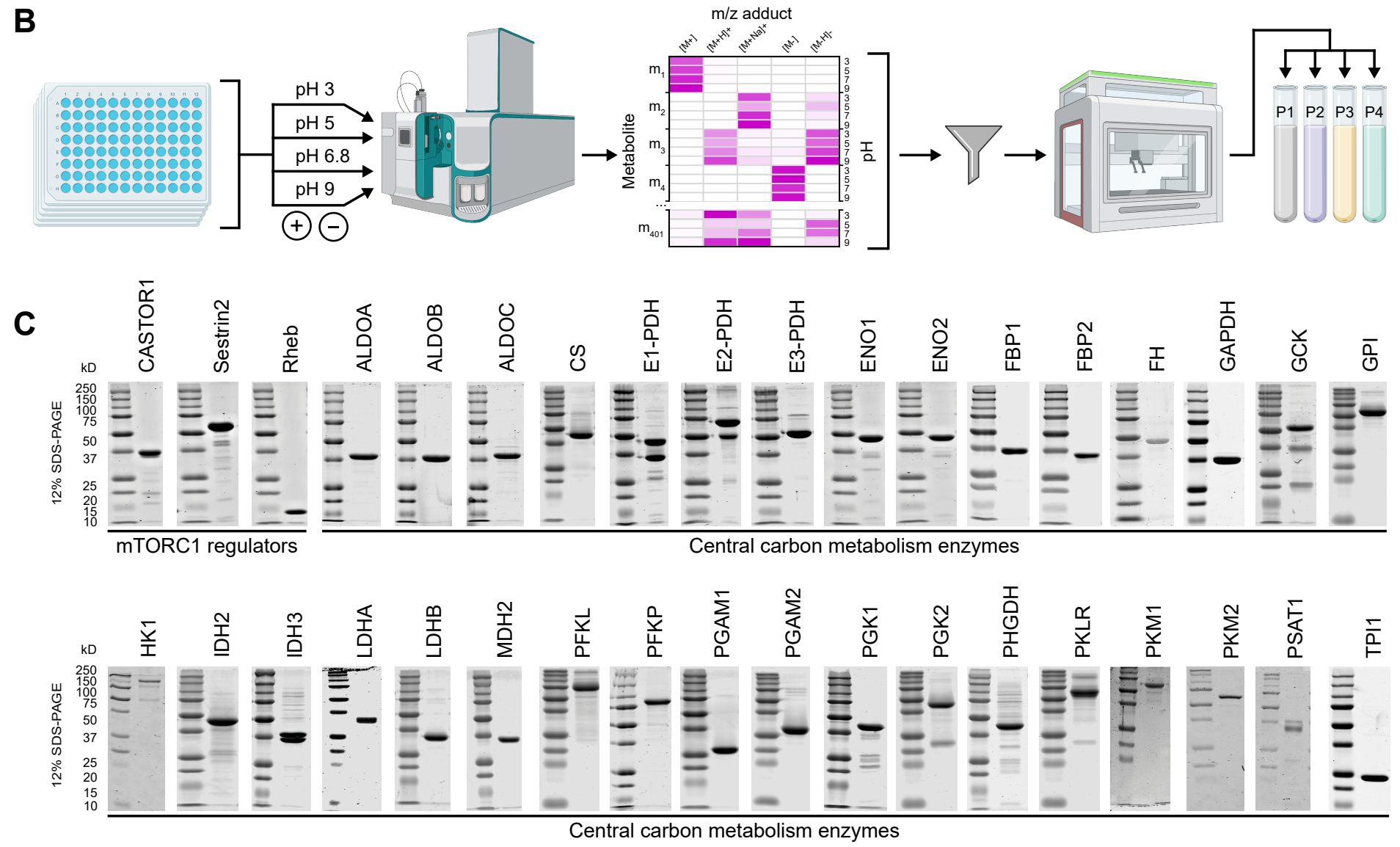
EDPxiv preprint doi: https://doi.org/10.1101/2021.08.28.458030; this version posted August 28, 2021. The copyright holder for this preprint Whan was not certified by peer review) is the author/funder, who has granted bioRxiv a license to display the preprint in perpetuity. It is made available under aCC-BY-NC-ND 4.0 International license.
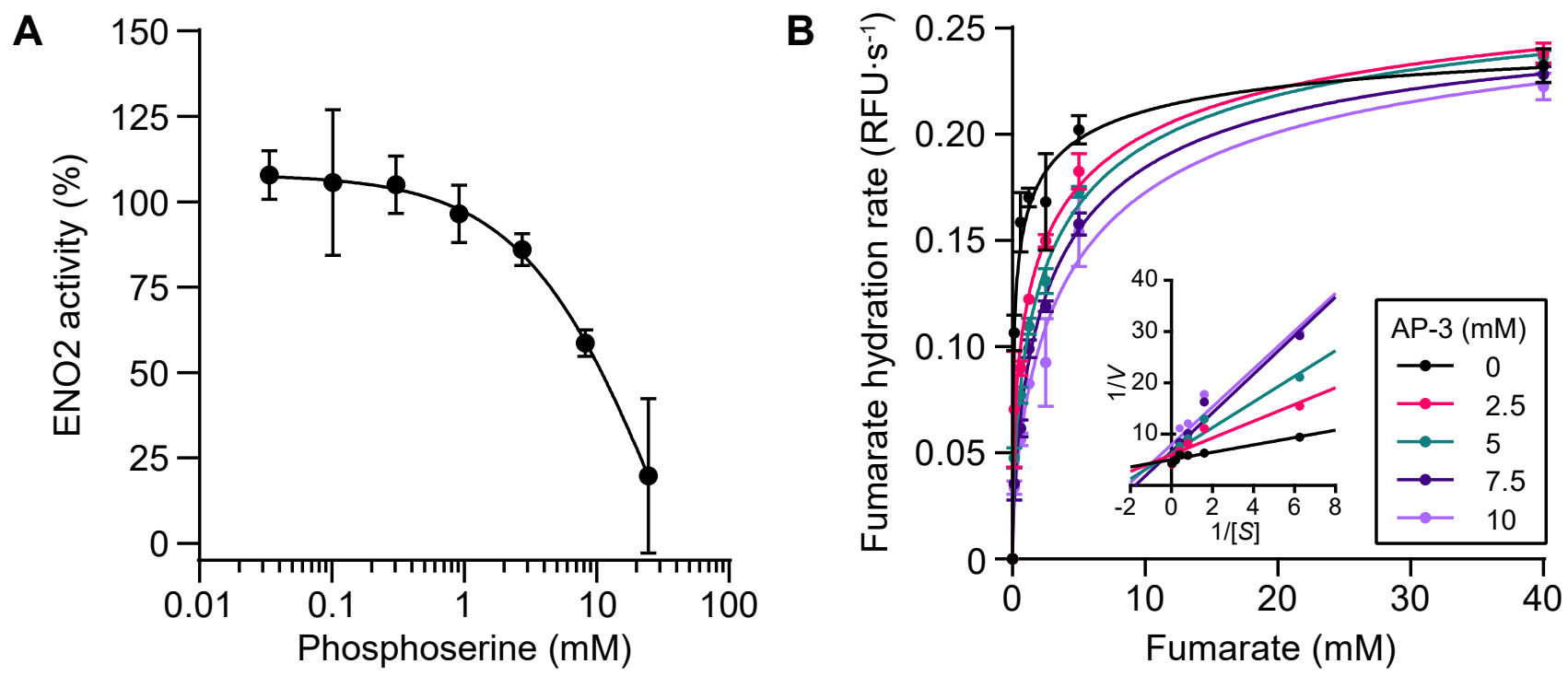
Eispxiv preprint doi: https://doi.org/10.1101/2021.08.28.458030; this version posted August 28, 2021. The copyright holder for this preprint 19. was not certified by peer review) is the author/funder, who has granted bioRxiv a license to display the preprint in perpetuity. It is made
available under aCC-BY-NC-ND 4.0 International license.

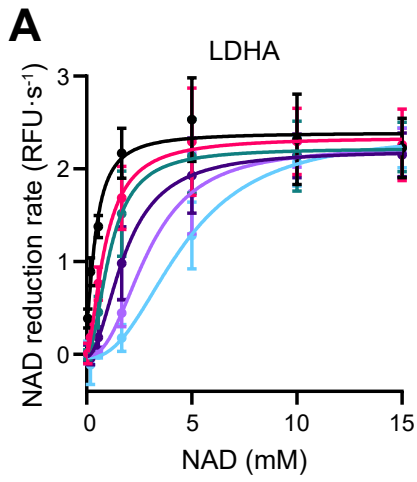

C
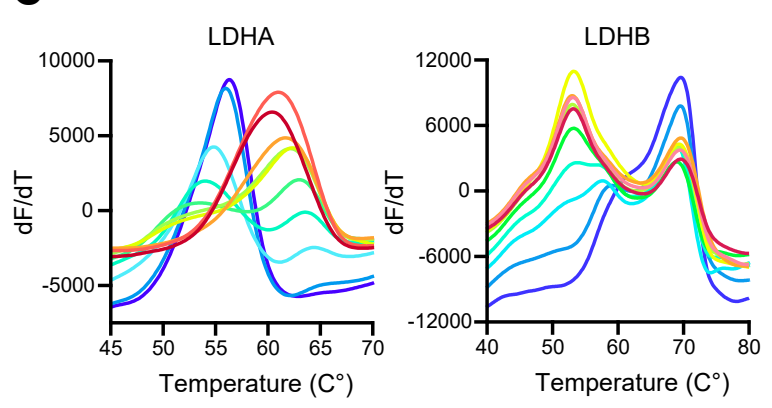

C16:0-CoA $(\mu \mathrm{M})$
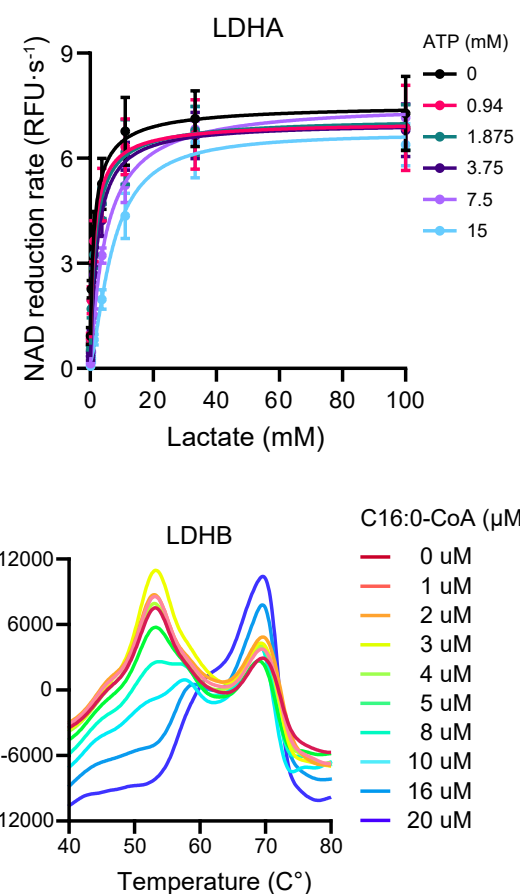

E

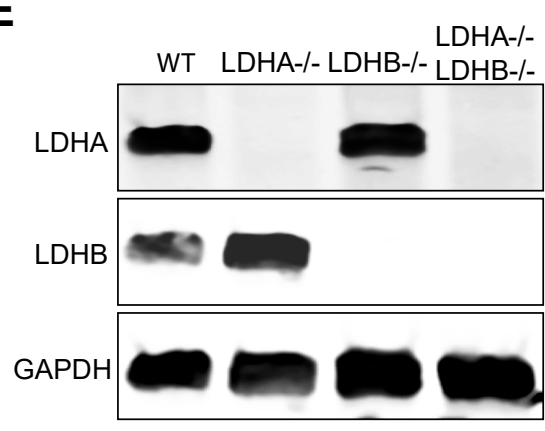

H

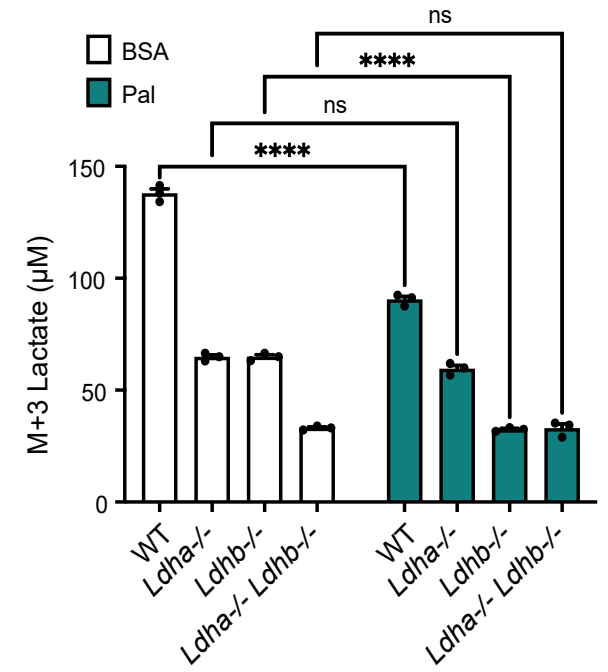

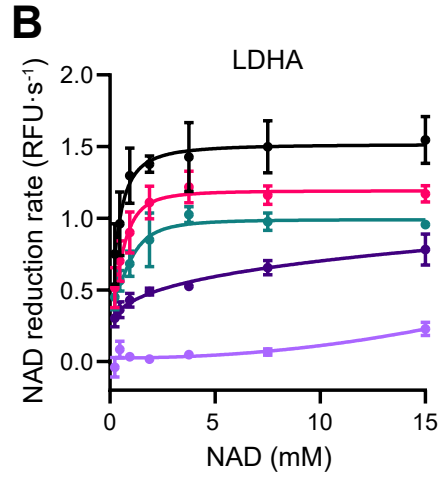

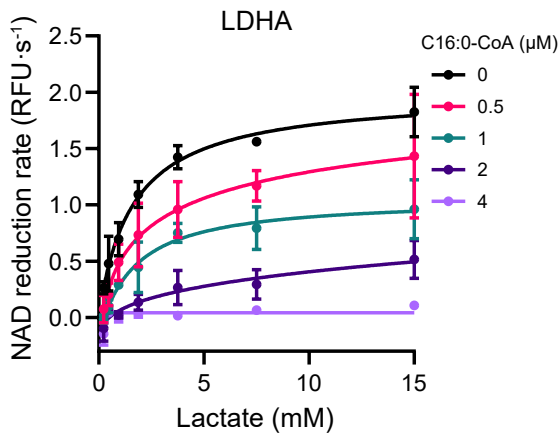

D

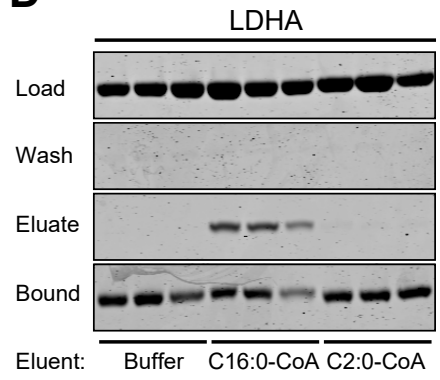

LDHB

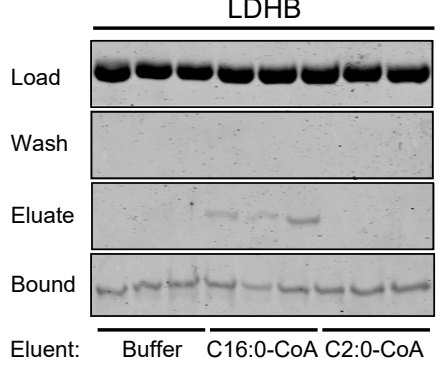

\section{F}

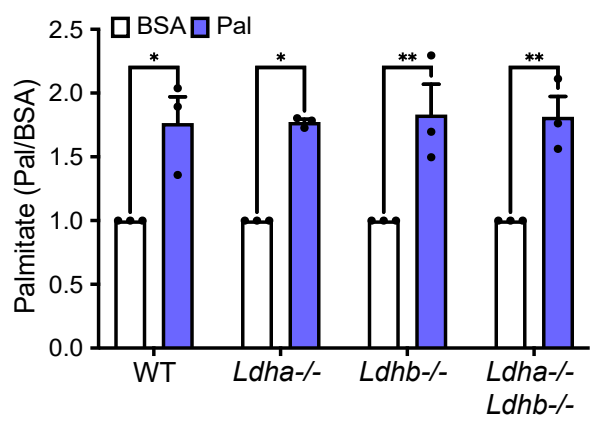

I

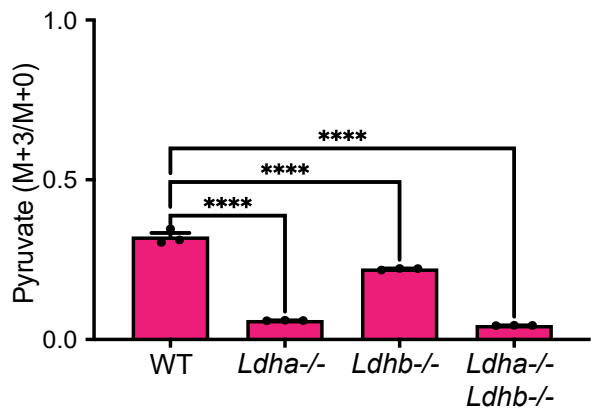

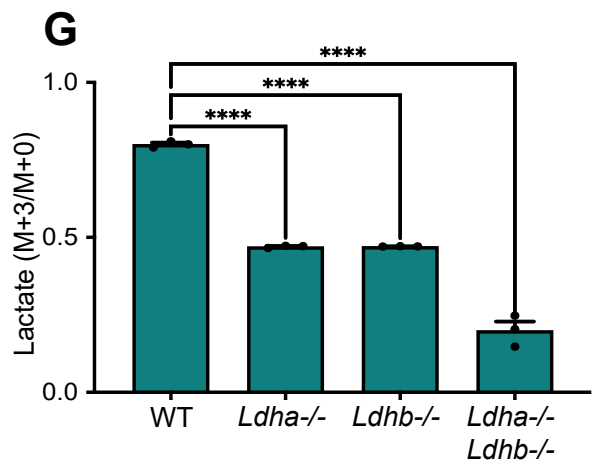

J

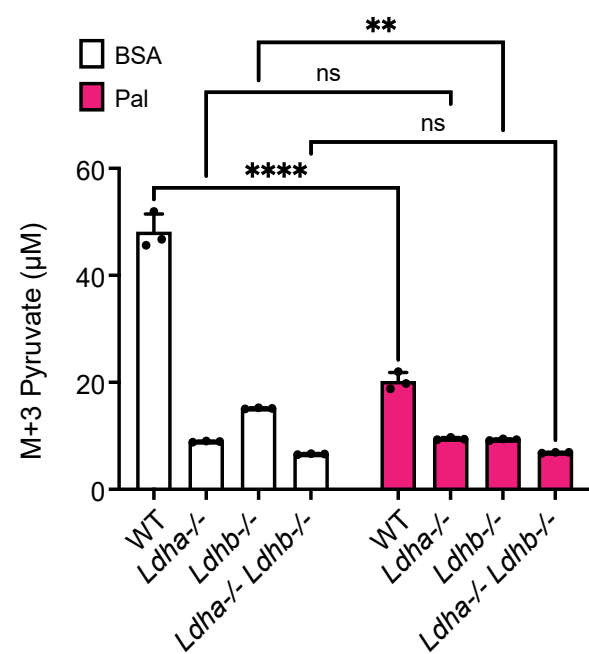


dioPxiv preprint doi: https://doi.org/10.1101/2021.08.28.458030; this version posted August 28, 2021. The copyright holder for this preprint 19. was not certified by peer review) is the author/funder, who has granted bioRxiv a license to display the preprint in perpetuity. It is made available under aCC-BY-NC-ND 4.0 International license.

A

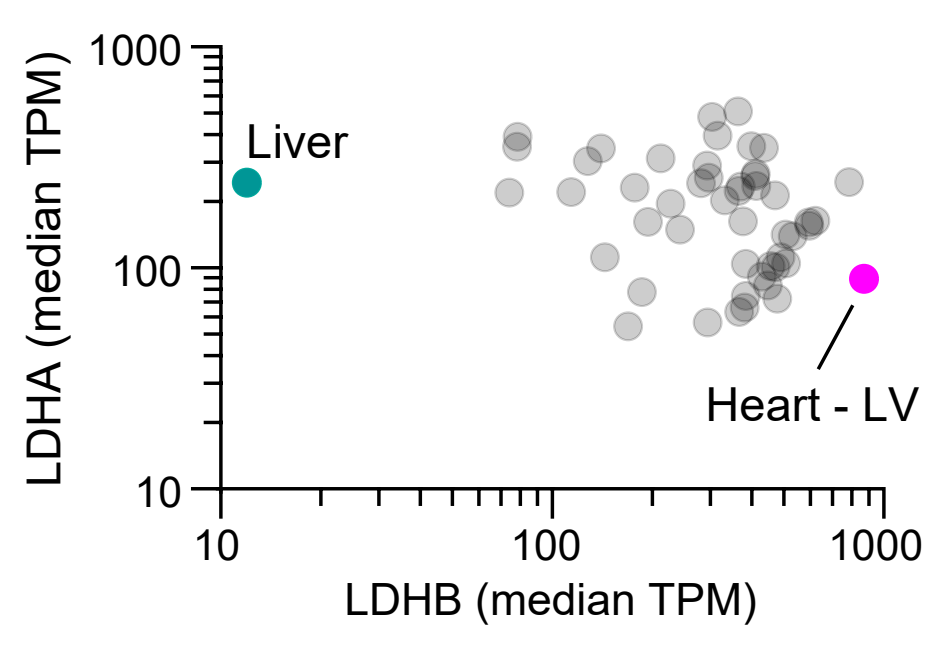

\section{B}

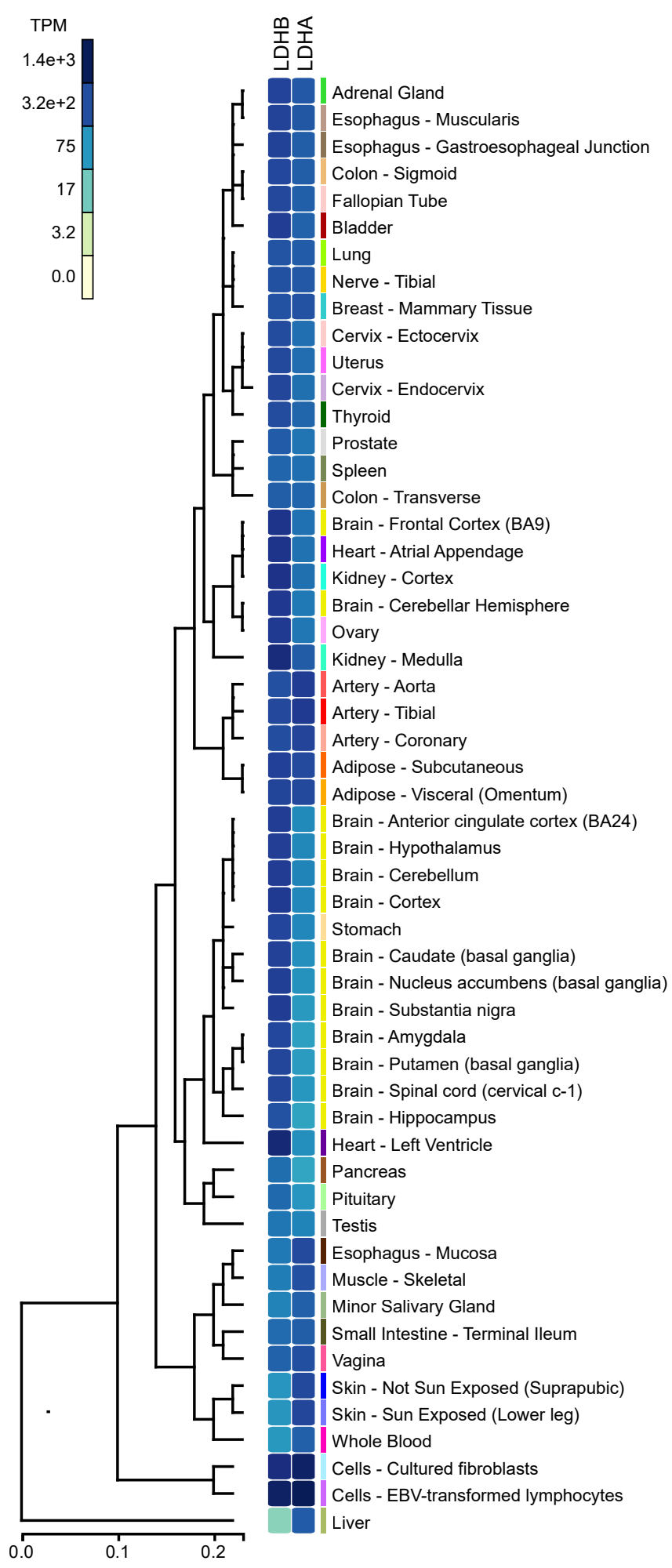




\section{Figure Legends}

730 Figure 1. MIDAS is a platform for the systematic discovery of protein-metabolite interactions.

731 (A) Biological systems are organized into domains of information (panes). Movement and interaction of 732 information in and through these domains underlies biological function (arrows). The MIDAS platform 733 provides protein-metabolite interactome (purple arrow) discovery. (B) The MIDAS platform is an equilibrium 734 dialysis tandem mass spectrometry approach. (Top) Purified proteins (cyan) are loaded into the protein 735 chamber $\left(P_{c}\right)$ and defined pools of metabolites into the metabolite chamber $\left(M_{c}\right)$, separated by a protein736 impermeable membrane. The system is incubated to relative equilibrium. (Bottom) Proteins are removed, 737 the $P_{c}$ and $M_{c}$ are sampled, and the relative abundance of metabolites from each chamber are quantified 738 using FIA-MS. Interactions between proteins and metabolites are observed as an increase (1, magenta) or 739 decrease (3, yellow) in integrated signal intensity in the $P_{c}$ relative to the $M_{c}$ (dotted peak). Metabolites that 740 have equal integrated signal intensity in the $P_{c}$ relative to the $M_{c}(2$, grey) are defined as non-interacting. 741 (C, D, E) The mTORC1 regulators CASTOR, Sestrin2, and Rheb were screened using MIDAS. Known 742 metabolite regulators are highlighted (magenta). Significant protein-metabolite interactions have a q-value $743<0.01$ (dotted line).

Figure 2. The protein-metabolite interactome of human central carbon metabolism.

746 (A) Heatmap representation of the MIDAS protein-metabolite interactomes of 33 enzymes in central carbon 747 metabolism. Heatmap values are the z-score log2(corrected fold change) for all metabolites in the MIDAS 748 metabolite library on a per protein basis. Clustering was performed by one minus Pearson correlation. 749 Positive (cyan) and negative (magenta) metabolite z-score log2(corrected fold change) have a maximum 750 and minimum cut-off of 10 and -10, respectively. (B, C, D) Excerpt examples of metabolite clustering from 751 (A). Colored bars (bottom) indicate the location of the extracted heatmaps from (A) (bottom). (E) 752 Multidimensional scaling (MDS) of 33 enzymes in central carbon metabolism based on their MIDAS protein753 metabolite interactomes. MDS distance values where generated from the z-score log2(corrected fold 754 change) for all metabolites in the MIDAS metabolite library on a per protein basis. (F) The top ten metabolite 755 sub-classes by total protein-metabolite interaction (PMI) count across 33 enzymes in human central carbon 756 metabolism. Metabolite sub-classes were modified from HMDB chemical taxonomy sub-class. (G and H) 757 Significant intra-pathway $(G)$ and inter-pathway $(H)$ interactions (colored lines) between metabolites (black 758 circles) and 33 enzymes in central carbon metabolism (orange boxes) detected by MIDAS (plots generated 759 in Electrum). Unique metabolites (dark grey circles), metabolite isoforms (light grey circles), metabolites not 760 present in the library (open circles). Significant protein-metabolite interactions have a q-value $<0.01$ and 761 are colored by increasing significance, light orange to red.

763 Figure 3. MIDAS reveals known and novel metabolite interactions with enzymes from human central 764 carbon metabolism. 
(A) Metabolite interactions with enolase 1 (ENO1, black) and enolase 2 (ENO2, pink). (B) Ligand-induced DSF melting point analysis of ENO1 (solid lines, solid circles) and ENO2 (dotted lines, open circles) with 2phosphoglycerate (2PG, black), phosphoserine (pSer, pink), serine (Ser, teal), phosphotyrosine (pTyr, purple), and phosphate (PO4, light purple). (C) X-ray crystal structure of the pSer-ENO2 complex (PDB $7 \mathrm{MBH}$ ). pSer (black box), phosphate ion (orange and red spheres), magnesium ion (green sphere), monomers within the ENO2 dimer (purple and teal). (D) Magnified view of the ENO2 active site with pSer (pink) or 2-phoshoglycerate (2PG) (grey) bound (2PG-ENO2, PDB 3UCC) (49). Secondary structure labeled in the pSer-ENO2 (purple) and 2PG-ENO2 (light grey) structures. (E) Metabolite interactions with fumarase (FH). (F) Ligand-induced DSF melting point analysis of FH with fumarate (Fum, black) and 2Amino-3-phosphonopropionic acid (AP-3, pink). (B and F) Line of best fit was determined using the specific binding and Hill slope equation from Prism 9. (G) X-ray crystal structure of the AP-3-FH complex (PDB 7LUB). AP-3 (black boxes), monomers within the FH tetramer (purple, yellow, teal, and light blue). (H) Magnified view of the FH active site with AP-3 (pink) or citrate (Cit, grey) bound (E. coli Cit-FH structure, light grey, PDB 1FUO) (13). Sidechains that coordinate the AP-3 interaction with FH are labeled and colored according to FH monomer from (G). (I) Metabolite interactions with glucose-6-phosphate isomerase (GPI). (J) Metabolite interactions with 6-Phosphofructokinase, platelet type (PFKP). (K) Metabolite interactions with glyceraldehyde-3-phosphate dehydrogenase (GAPDH). (L) Metabolite interactions with pyruvate kinase M2 (PKM2). (M) Metabolite interactions with fructose-1,6-bisphosphatase 1 (FBP1, black) and fructose-1,6-bisphosphatase 2 (FBP2, pink). (N) Metabolite interactions with phosphoglycerate mutase 1 (PGAM1, black) and phosphoglycerate mutase 2 (PGAM2, pink). (A, E, I- N) Volcano plots generated from MIDAS PMI data. Specific metabolites are labeled. Stars indicate a previously known human PMI primarily sourced from BRENDA (https://www.brenda-enzymes.org/index.php). Significant protein-metabolite interactions have a q-value $<0.01$ (dotted line).

Figure 4. ATP and long-chain acyl-CoAs inhibit lactate dehydrogenase in an isoform-specific manner.

(A) Metabolite interactions with lactate dehydrogenase A (LDHA, black) and lactate dehydrogenase B (LDHB, pink). Volcano plots generated from MIDAS PMI data. Specific metabolites are numbered and

labeled. Stars indicate a previously known human PMI primarily sourced from BRENDA 794 (https://www.brenda-enzymes.org/index.php). Significant protein-metabolite interactions have a q-value < 0.01 (dotted line). (B) Metabolite classes that interact with LDHA and LDHB from (A) (nicotinamides and 796 dinucleotides, purple; adenosine nucleotide derivatives, pink; coenzyme A derivatives, yellow; keto acids, 797 teal). (C) Ligand-induced DSF melting point analysis of LDHA (solid lines, filled circles) and LDHB (dotted 798 lines, open circles) with adenosine triphosphate (ATP, black), adenosine diphosphate (ADP, light purple), 799 adenosine monophosphate (AMP, teal), and nicotinamide adenine dinucleotide (NAD, pink). Apparent 800 dissociation constant $\left(\mathrm{Kd}_{\mathrm{app}}\right)$ was determined from triplicate experiments using the specific binding and Hill 801 slope equation from Prism 9. Mean \pm SD is plotted from triplicate experiments. (D) Enzyme activity of LDHA 
802 (solid lines, filled circles) and LDHB (dotted lines, open circles) treated with ATP (black), ADP (light purple), or AMP (teal). (E and F) Enzyme activity of LDHA or LDHB treated with coenzyme A (CoA, grey), acetyl-

804 CoA (C2:0-CoA, cyan), butyryl-CoA (C4:0-CoA, light pink), octanoyl-CoA (C8:0-CoA, light purple), lauroyl-

805 CoA (C12:0-CoA, black), palmitoyl-CoA (C16:0-CoA, teal), oleoyl-CoA (C18:1-CoA, pink), and saturated

806 arachidonoyl-CoA (C20:0-CoA, purple). (D - F) Half maximal inhibitory concentration (IC50) was determined

807 from triplicate experiments using Prism 9; ND, not determined. Mean \pm SD is plotted from triplicate

808 experiments. (G) Schematic of $\left[\mathrm{U}^{13} \mathrm{C}_{6}\right]$-glucose metabolism in cells treated with palmitate-conjugated BSA.

809 Pyruvate, Pyr; lactate, Lac; IC, intracellular; EC, extracellular. (H) Fold change of extracellular [U $\left.{ }^{13} \mathrm{C}_{3}\right]-$

810 lactate collected from the growth media of the indicated $\mathrm{H} 9 \mathrm{c} 2$ cell lines in response to treatment with

811 palmitate-conjugated BSA (Pal) relative to BSA control. (I) Schematic of $\left[\mathrm{U}^{13} \mathrm{C}_{3}\right]$-lactate metabolism in cells

812 treated with palmitate-conjugated BSA. Pyruvate, Pyr; lactate, Lac; IC, intracellular; EC, extracellular. (J)

813 Fold change of intracellular $\left[\mathrm{U}^{13} \mathrm{C}_{3}\right]$-pyruvate in indicated $\mathrm{H} 9 \mathrm{c} 2$ cell lines in response to treatment with

814 palmitate-conjugated BSA (Pal) relative to BSA control. ( $\mathbf{H}$ and $\mathbf{J}$ ) Experiments were performed in triplicate

815 and mean \pm SD is displayed. A students t-test was performed between Pal and BSA samples $(p<0.005$,

$81{ }^{* *} ; p<0.00005,{ }^{* * * *}$.

\section{Supplemental Figure Legends}

819 Figure S1. MIDAS metabolite library construction and validation and screened proteins.

820 (A) The MIDAS metabolite library overlaid on KEGG human metabolic pathways

821 (https://www.genome.jp/kegg/pathway/map/map01100.html). Human pathways, colored; non-human

822 pathways, light grey. Metabolites from the MIDAS metabolite library, black spheres. (B) FIA-MS scouting

823 method to determine optimal MIDAS metabolite pools. Metabolites from the MIDAS metabolite library were

824 arrayed across five 96-well plates in water. Each metabolite was individual analyzed at mobile phase $\mathrm{pH} 3$,

$8255,6.8$, and 9, in positive and negative mode, by FIA-MS. For each metabolite $\left(m_{1}-m_{401}\right)$ analyzed by FIA-

826 MS, accurate mass was verified and optimal signal was determined from the integrated area under the

827 curve for the extracted ion chromatogram of each metabolite adduct, mobile phase $\mathrm{pH}$, and polarity

828 (increasing FIA-MS signal, white to magenta Heatmap). The optimal FIA-MS signal conditions of each

829 metabolite were manually filtered and binned to program an automated liquid handling method to construct

830 the MIDAS metabolite pools (P1, P2, P3, and P4) according to the specific conditions of metabolite analysis

831 by FIA-MS. (C) SDS-PAGE analysis of the purified proteins analyzed by MIDAS. mTORC1 regulators and

832 the enzymes from central carbon metabolism are labeled.

834 Figure S2. Enzymatic activities of enolase and fumarase with interacting metabolites.

835 (A) Activity of enolase, expressed as a percentage of vehicle control, was determined in the presence of 836 varying concentrations of phosphoserine (pSer) using a coupled enzyme kinetic assay with pyruvate 837 kinase/lactate dehydrogenase. The experiment was performed in triplicate and the mean \pm SD are plotted. 
(B) Relative fumarate hydration rate by fumarase was determined in the presence of varying concentrations of substrate, fumarate, and the inhibitor, 2-amino-3-phosphonopropionic acid (AP-3, colored) using a malate dehydrogenase coupled enzyme assay. (Inset) Lineweaver-Burk plot demonstrating competitive inhibition. The experiment was performed in triplicate and the mean \pm SD are plotted.

Figure S3. Lactate dehydrogenase interacts with and is inhibited by nucleotides and long-chain acyl-CoA.

(A) Relative NAD reduction rate by LDHA was determined in the presence of varying concentrations of NAD (cofactor) or lactate (substrate), and ATP (colored) using a lactate dehydrogenase enzyme assay. The experiments were performed in triplicate and the mean \pm SD are plotted. (B) Relative NAD reduction rate by LDHA was determined in the presence of varying concentrations of NAD (cofactor) or lactate (substrate), and palmitoyl-CoA (C16:0-CoA) (colored) using a lactate dehydrogenase enzyme assay. The experiments were performed in triplicate and the mean \pm SD are plotted. (C) LDHA and LDHB were analyzed by PROTEOSTAT DSF in the presence of increasing concentrations of C16:0-CoA (colored). $\mathrm{dF} / \mathrm{dT}$ was determined as a function of temperature. Representative experiments from triplicate experiments are presented. (D) Palmitoyl-CoA-Agarose pull-down assay with LDHA or LDHB treated with buffer control, palmitoyl-CoA (C16:0-CoA), or acetyl-CoA (C2:0-CoA) (Eluent). Protein input (Load), post$5 x$ wash (Wash), concentrated supernatant post-eluent treatment (Eluate), protein bound to palmitoyl-CoAagarose beads post-eluent treatment (Bound). The experiment was performed in triplicate. (E) Representative immunoblot of LDHA and LDHB in the indicated H9c2-derived cell lines. (F) Fold change of intracellular palmitate in Ldha-/-, Ldhb-/-, or Ldha-/-;Ldhb-/- H9c2 cell lines in response to treatment with palmitate-conjugated BSA (Pal) relative to BSA vehicle control (BSA). (G) Changes in ${ }^{13} \mathrm{C}$ enrichment of extracellular lactate in Ldha-/-, Ldhb-/-, or Ldha-/-;Ldhb-/- H9c2 cell lines in response to treatment with palmitate-conjugated BSA. (H) Concentration of ${ }^{13} \mathrm{C}$-labelled extracellular lactate in Ldha-/-, Ldhb-/-, or Ldha-/-;Ldhb-/- H9c2 cell lines in response to BSA vehicle control (BSA) or palmitate-conjugated BSA (Pal) treatment. (I) Changes in intracellular ${ }^{13} \mathrm{C}$ enrichment of pyruvate in Ldha-/-, Ldhb-/-, or Ldha-/-;Ldhb-/$\mathrm{H} 9 \mathrm{c} 2$ cell lines in response to treatment with palmitate-conjugated BSA. (J) Concentration of intracellular ${ }^{13}$ C-labelled pyruvate in Ldha-/-, Ldhb-/-, or Ldha-/-;Ldhb-/- H9c2 cell lines in response to BSA vehicle control (BSA) or palmitate-conjugated BSA (Pal) treatment. $(\mathbf{F}-\mathbf{G})$ All experiments were performed in triplicate. Data are presented as mean \pm SD. $* p<0.05, * * p<0.01, * * * p<0.001, * * * * p<0.0001$, transcripts per million (TPM) for LDHA and LDHB. Note logarithmic axes. (B) Heatmap depicting median TPM for LDHA and LDHB across human tissues. Data obtained through GTEx Portal. 
874 Table S1. Phosphoserine-ENO2 data collection and refinement statistics.

\begin{tabular}{|c|c|}
\hline Wavelength $(\AA)$ & 1.00000 \\
\hline Resolution range $(\AA)$ & $39.89-2.10(2.175-2.10)^{*}$ \\
\hline Space group & $P 2{ }_{1}{ }_{1} 2_{1}$ \\
\hline Unit cell $(\AA \circ)$ & 68.0498108 .38117 .929 \\
\hline$\left(^{\circ}\right)$ & $90,90,90$ \\
\hline Total reflections & $2203993(188723)^{*}$ \\
\hline Unique reflections & $50504(4644)^{*}$ \\
\hline Multiplicity & $43.6(38.5)^{\star}$ \\
\hline Completeness (\%) & $96.58(90.06)^{*}$ \\
\hline Mean I/sigma(I) & $20.52(1.92)^{\star}$ \\
\hline Wilson B-factor $\left(\AA^{2}\right)$ & 25.70 \\
\hline R-merge & $0.9095(3.46)^{*}$ \\
\hline R-meas & $0.92(3.508)^{*}$ \\
\hline R-pim & $0.1353(0.5575)^{*}$ \\
\hline $\mathrm{CC} 1 / 2$ & $0.931(0.539)^{\star}$ \\
\hline $\mathrm{CC}^{*}$ & $0.982(0.837)^{*}$ \\
\hline Reflections used in refinement & $49880(4573)^{*}$ \\
\hline Reflections used for R-free & $2482(226)^{*}$ \\
\hline R-work & $0.1802(0.2715)^{*}$ \\
\hline R-free & $0.2348(0.3264)^{*}$ \\
\hline CC(work) & $0.849(0.706)^{\star}$ \\
\hline $\mathrm{CC}($ free $)$ & $0.841(0.649)^{*}$ \\
\hline Number of non-hydrogen atoms & 7008 \\
\hline macromolecules & 6666 \\
\hline ligands & 44 \\
\hline solvent & 298 \\
\hline Protein residues & 870 \\
\hline RMS(bonds) $(\AA)$ & 0.007 \\
\hline RMS(angles) $\left(^{\circ}\right)$ & 0.88 \\
\hline Ramachandran favored (\%) & 96.88 \\
\hline Ramachandran allowed (\%) & 2.89 \\
\hline Ramachandran outliers (\%) & 0.23 \\
\hline Rotamer outliers (\%) & 1.85 \\
\hline Clashscore & 4.72 \\
\hline Average B-factor $\left(\AA^{2}\right)$ & 30.34 \\
\hline macromolecules & 30.16 \\
\hline ligands & 43.30 \\
\hline solvent & 32.47 \\
\hline
\end{tabular}

$875{ }^{*}$ Statistics for the highest-resolution shell are shown in parentheses. 
876 Table S2. AP-3-fumarase data collection and refinement statistics.

\begin{tabular}{|c|c|}
\hline Wavelength $(\AA \dot{A})$ & 1.323630 \\
\hline Resolution range $(\AA)$ & $49.80-2.15(2.20-2.15)^{*}$ \\
\hline Space group & P 6522 \\
\hline Unit cell $(\AA)$ & $190.90,116.24,190.90$ \\
\hline$\left(^{\circ}\right)$ & $90,120,90$ \\
\hline Total reflections & $2660677(178767)^{*}$ \\
\hline Unique reflections & $67921(4478)^{*}$ \\
\hline Multiplicity & $39.2(39.9)^{*}$ \\
\hline Completeness (\%) & $100(100)^{*}$ \\
\hline Mean I/sigma(I) & $15.0(1.1)^{*}$ \\
\hline Wilson B-factor & 43.3 \\
\hline R-merge & $0.25(4.82)^{*}$ \\
\hline R-meas & $0.26(4.95)^{*}$ \\
\hline R-pim & $0.056(1.088)^{*}$ \\
\hline $\mathrm{CC} 1 / 2$ & $0.999(0.458)^{*}$ \\
\hline $\mathrm{CC}^{*}$ & - \\
\hline Reflections used in refinement & 67877 \\
\hline Reflections used for R-free & 3399 \\
\hline R-work & $0.1858(0.3100)^{*}$ \\
\hline R-free & $0.2088(0.3300)^{*}$ \\
\hline CC(work) & $0.965(0.6406)^{*}$ \\
\hline CC(free) & $0.955(0.6746)^{*}$ \\
\hline Number of non-hydrogen atoms & 7191 \\
\hline macromolecules & 6847 \\
\hline ligands & 44 \\
\hline solvent & 300 \\
\hline Protein residues & 922 \\
\hline RMS deviations (bonds) & 0.0031 \\
\hline RMS deviations (angles) & 1.266 \\
\hline Ramachandran favored (\%) & 96.82 \\
\hline Ramachandran allowed (\%) & 3.18 \\
\hline Ramachandran outliers (\%) & 0.00 \\
\hline Rotamer outliers (\%) & 0.84 \\
\hline Clashscore & 2.92 \\
\hline Average B-factor & 52.00 \\
\hline macromolecules & 52.09 \\
\hline ligands & 56.03 \\
\hline solvent & 50.97 \\
\hline
\end{tabular}

$877{ }^{*}$ Statistics for the highest-resolution shell are shown in parentheses. 
bioRxiv preprint doi: https://doi.org/10.1101/2021.08.28.458030; this version posted August 28, 2021. The copyright holder for this preprint (which was not certified by peer review) is the author/funder, who has granted bioRxiv a license to display the preprint in perpetuity. It is made available under aCC-BY-NC-ND 4.0 International license.

879 Other Supplementary Materials for this manuscript include the following:

880 Data S1. MIDAS metabolite library

881 Data S2. FIA-MS properties of MIDAS metabolites

882 Data S3. MIDAS proteins

883 Data S4. MIDAS protein-metabolite interactions 